\title{
A pattern-based approach to the interpretation of skeletal muscle biopsies
}

\author{
Chunyu Cai ${ }^{1} \cdot$ Douglas C. Anthony $\mathbb{B}^{2} \cdot$ Peter Pytel $\mathbb{D}^{3}$
}

Received: 23 July 2018 / Revised: 24 September 2018 / Accepted: 25 September 2018 / Published online: 6 November 2018

(c) United States \& Canadian Academy of Pathology 2018

\begin{abstract}
The interpretation of muscle biopsies is complex and provides the most useful information when integrated with the clinical presentation of the patient. These biopsies are performed for workup of a wide range of diseases including dystrophies, metabolic diseases, and inflammatory processes. Recent insights have led to changes in the classification of inflammatory myopathies and have changed the role that muscle biopsies have in the workup of inherited diseases. These changes will be reviewed. This review follows a morphology-driven approach by discussing diseases of skeletal muscle based on a few basic patterns that include cases with (1) active myopathic damage and inflammation, (2) active myopathic damage without associated inflammation, (3) chronic myopathic changes, (4) myopathies with distinctive inclusions or vacuoles, (5) biopsies mainly showing atrophic changes, and (6) biopsies that appear normal on routine preparations. Each of these categories goes along with certain diagnostic considerations and pitfalls. Individual biopsy features are only rarely pathognomonic. Establishing a firm diagnosis therefore typically requires integration of all of the biopsy findings and relevant clinical information. With this approach, a muscle biopsy can often provide helpful information in the diagnostic workup of patients presenting with neuromuscular problems.
\end{abstract}

\section{Introduction}

Patients with neuromuscular disease often present with symptoms like weakness, episodic muscle cramping, myalgias, or rhabdomyolysis. Skeletal muscle biopsies are performed as a part of the diagnostic workup for certain patients with neuromuscular diseases [1] for whom the differential diagnosis may include the following broad disease categories: (a) Autoimmune inflammatory conditions; (b) Genetic diseases including muscular dystrophies and congenital myopathies; (c) Toxic or drug-induced myopathies; (d) Metabolic or endocrine diseases; (e) Other

Peter Pytel

peter.pytel@uchospitals.edu

1 Department of Pathology, UT Southwestern Medical Center, Dallas, TX, USA

2 Departments of Pathology and Laboratory Medicine, and Neurology, Alpert Medical School of Brown University, Providence, RI, USA

3 Department of Pathology, University of Chicago, Chicago, IL, USA rare conditions. It is difficult to assess how much the results of a muscle biopsy contribute to the management of a patient because the yield depends on the setting and the clinical question the biopsy was intended to answer [2-6]. In some instances, even a "negative" biopsy may be regarded as informative to the clinical team, if it eliminates some differential diagnostic possibilities. Overall the utility is strongly influence by the proper planning, execution and processing of the biopsy. The optimal planning of the biopsy may involve the primary treating physician-most often a neurologist or rheumatologist - the surgeon, the pathologist, and sometimes the radiologist. Clinical presentation, physical exam, electromyography (EMG) as well as magnetic resonance imaging (MRI) may all be considered when deciding which muscle will provide the most useful information.

The interpretation of muscle biopsies is often challenging because morphologic features are only rarely sufficient to assign a muscle biopsy to any of the classes of muscle diseases mentioned above let alone a specific disease entity. Instead, it is usually the combination of multiple different changes that may suggest a diagnosis. Furthermore, the interpretation of a biopsy can be more precise if the clinical context as well as the histomorphologic findings are 


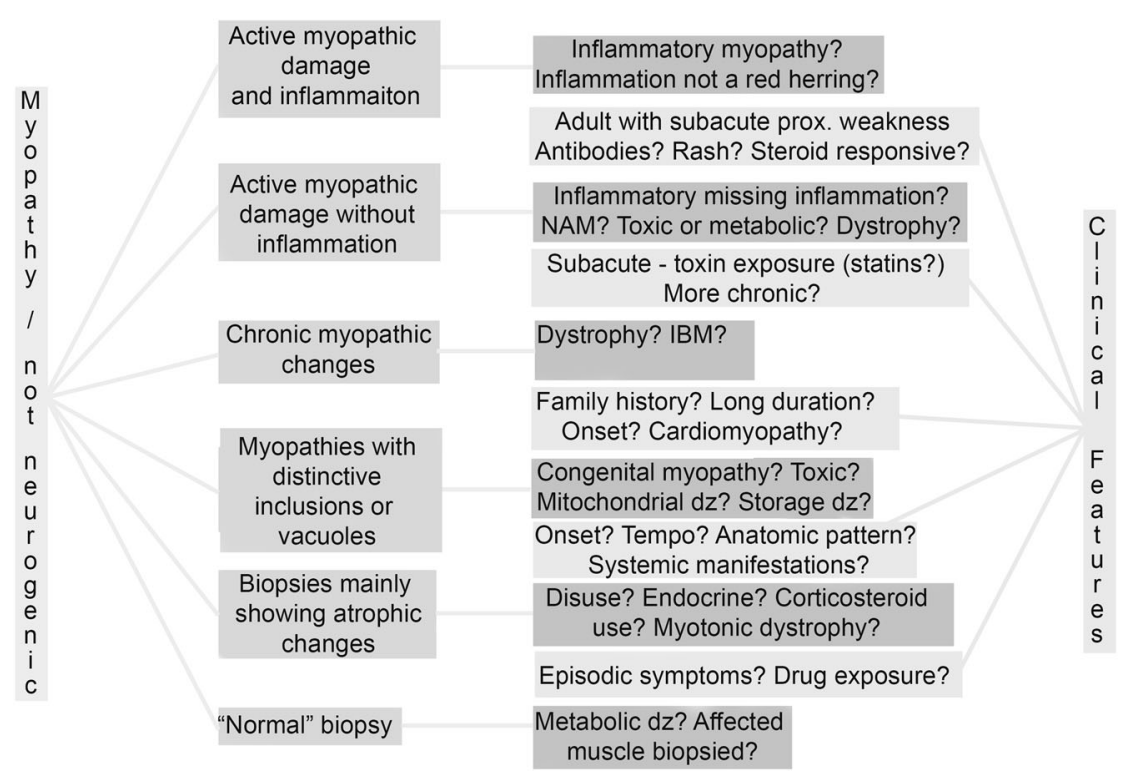

Fig. 1 Diagram of pattern-based approach to muscle biopsies. This diagram illustrates the pattern-based approach discussed in this review and sketches out how clinical information can be integrated into formulating a differential diagnosis. Most biopsies may be thought of as falling into six main morphologic patterns of myopathic findings (once a neurogenic etiology is excluded). Illustrated in light orange, each of these patterns goes along with a distinct set of diagnostic considerations. A biopsy showing inflammation, for example, is most often suggestive of an inflammatory myopathy, but may also be seen in other conditions, which should be addressed pathologically or in the context of the clinical presentation. Correlation with clinical information (green) including systemic disease manifestations, temporal pattern, autoantibodies, and steroid responsiveness may be extremely helpful. A biopsy with active myopathic damage that lacks inflammatory infiltrates has a wide differential diagnosis that may include

considered and integrated. This review organizes diseases of skeletal muscle based on a few recurring morphologic patterns to reflect the thought process of a pathologist evaluating cellular histopathology. As a result, it does not follow the didactic approach based on pathophysiology that many textbooks utilize.

Weakness may be the result of injury of the upper or lower motor neuron resulting in denervation, or it may be the result of injury of the myocyte, resulting in a primary myopathic process. Morphologic changes that point to denervation of muscle include angulated atrophic fibers, grouped atrophy, and fiber-type grouping. These findings reflect a loss of motor innervation of the myocyte, and occur as a result of injury of the motor neuron or its axonal processes. They are sometimes referred to as "denervation atrophy", or less specifically as "neurogenic atrophy".

Once a neurogenic process has been excluded, most muscle biopsy specimens may be categorized into one of the following six descriptive categories, defined by the findings on histologic sections stained with hematoxylin and eosin (H\&E) and the modified Gomori trichrome stain (Fig. 1): (I) Active myopathic damage and inflammation; necrotizing autoimmune myopathy (NAM), toxic muscle damage, or a metabolic disease. The clinical information may again help to narrow this list down and allow judicious use of additional special stains and ancillary testing. Chronic myopathic changes are often a feature of an inherited process like a muscular dystrophy but may also be present in acquired diseases like sporadic inclusion-body myositis (IBM). In biopsies showing distinctive inclusions or vacuoles, the diagnosis depends on the nature of the intracellular material forming theses and the clinical context. Atrophic changes may be present in many different disease processes but may be a prominent finding in endocrine disorders, disuse, prior steroid therapy, or myotonic dystrophy. Details on the pattern of atrophy and the clinical history may be helpful in defining the etiology of an otherwise often rather non-specific morphologic change

(II) Active myopathic damage without associated inflammation; (III) Chronic myopathic changes; (IV) Myopathies with distinctive inclusions or vacuoles; (V) Biopsies mainly showing atrophic changes; (VI) Biopsies that appear normal. The term active myopathic damage refers to the presence of necrotic myofibers and regenerating myofibers (Fig. 2). In this context, inflammation refers to the presence of mononuclear inflammatory cells (i.e., lymphocytes, plasma cells, or macrophages) in the perimysial or endomysial connective tissue but does not include macrophages associated with myofiber necrosis (known as myophagocytosis). Chronicity is usually evident in a muscle biopsy in the form of endomysial fibrosis and fatty replacement (Fig. 2). Often marked variation in fiber size, prominently increased internal nuclei, and fiber splitting accompany these chronic changes.

Each of these six broad categories is associated with certain diagnostic considerations that can be further interrogated by additional special stains and ancillary testing depending on the clinical setting. In addition, each has unique diagnostic pitfalls to avoid. This review outlines an approach to examining muscle biopsy specimens, addressing them from the 

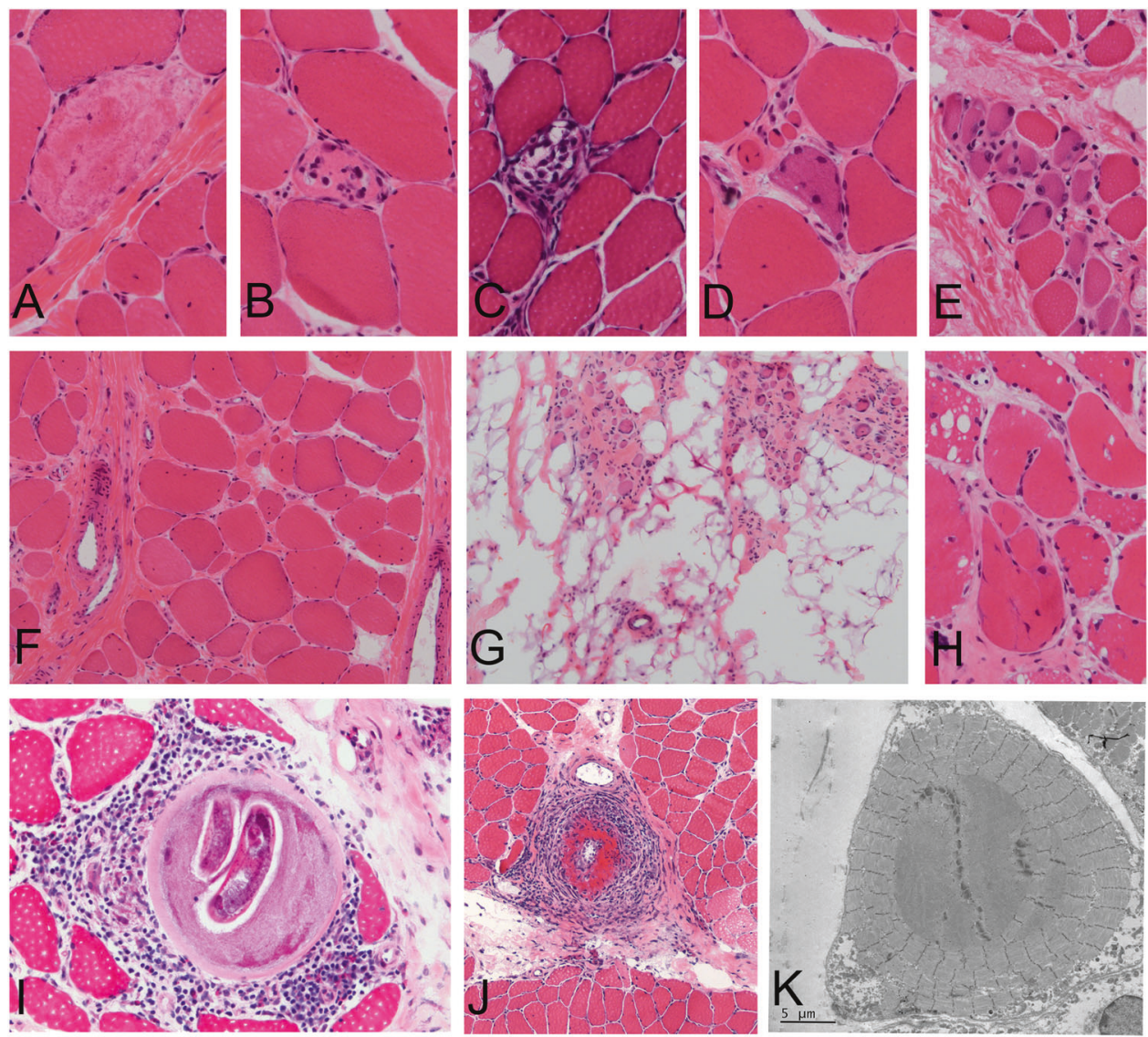

Fig. 2 Illustration of miscellaneous morphologic changes encountered in muscle biopsies. Muscle fiber necrosis and regeneration $(\mathbf{a}-\mathbf{e})$. Individual myofibers can undergo necrosis and regeneration that is usually assumed to represent a segmental process. Individual fiber necrosis and regeneration is a hallmark feature present in many myopathies. In the early phase, the cytoplasm assumes an often more pale uneven appearance with more amorphous irregular texture (a). Subsequently, immune cells begin to infiltrate the necrotic myofiber segment (b) in a process described as "myophagocytosis". Eventually only the hull of the fiber remains as a bag filled with immune cells (c). Regenerating myofibers are characterized by a more basophilic cytoplasm reflective of increased mRNA content and enlarged nuclei that often contain distinctly visible nucleoli (d). Clustered regenerating myofibers in a case of Duchenne muscular dystrophy (e). Features of disease chronicity $(\mathbf{f}-\mathbf{h})$. Settings that go along with muscle damage outpacing regenerative potential lead to chronic remodeling in the form of endomysial fibrosis and fatty replacement. In mild cases, subtle collagen deposition is seen between the myofibers within a fascicle (Becker dystrophy, f). In more extensive cases, chronic remodeling can lead to extensive replacement of muscle tissue by fat and fibrosis (central core disease, g). Other features typically encountered in chronic myopathies include marked variation in myofibers size with atrophic fibers but often also hypertrophied fibers. The latter sometimes exhibit features of fiber splitting (h) that may at times go along with displacement of capillaries into the resulting clefts. Inflammatory infiltrates (i, j). Inflammatory infiltrates in the form of endomysial or perimysial infiltrates most commonly comprised of mononuclear inflammatory cells are a typical feature of inflammatory myopathies. They can, however, also be present in a number of other settings including some dystrophies, infections (trichinosis, i), focal myositis, or vasculitis (j). Ring fibers (k) are a fairly non-specific feature that is often present in cases of myotonic dystrophy point of view of these six basic morphologic patterns. Such an approach runs the risk of oversimplifying a complex topic, but offers a guide especially for those that are only occasionally asked to review muscle biopsy slides or those that communicate results from a specialty laboratory to local physicians.

\section{Section I: Active myopathic damage and inflammation}

The combination of active myopathic changes and inflammatory infiltrates in a muscle biopsy is typical for an active inflammatory myopathy. There are, however, notable exceptions. On the one hand, some immune-mediated myopathies lack distinctive inflammatory infiltrates and may instead present with a morphologic picture of "necrotizing autoimmune myopathy" discussed below in Section II. On the other hand, inflammation may sometimes be present in myopathies that are not immune-mediated, as discussed further in the Section III on muscular dystrophies.

Inflammatory myopathies are a complex group of entities characterized by variable temporal and anatomic patterns of muscle involvement, differences in associated systemic manifestations, and association with certain autoantibodies 
(Table 1) [7-9]. A number of classification systems are in current use. In 1975, Bohan and Peter emphasized the separation of dermatomyositis from polymyositis in a classification that hinged in part on the presence or absence of skin involvement [10, 11]. The subsequent integration of the diagnosis of sporadic inclusion-body myositis (sIBM, often simply referred to as "IBM") led to a long-standing triad of inflammatory myopathies that is still reflected in many textbooks: polymyositis, dermatomyositis, and sporadic inclusion-body myositis. Alternative classification systems have been proposed which vary in the emphasis they place on clinical features, serologic biomarkers, and muscle biopsy results [8, 12-17]. The use of different criteria and different terminologies make the inflammatory myopathies a challenging topic. The European Neuromuscular Consortium (ENMC) 2003 international workshop [14] and its 2014 update [18] represents a consensus approach. It is very similar to the classifications proposed by Allenbach et al. [8] and Suzuki et al. [19]. Our approach to inflammatory myopathies will largely follow that outlined in these publications with brief references to the terminology used by other investigators. Morphologic features to consider include the distribution of myofiber damage and atrophy, the pattern and distribution of inflammatory infiltrates, features of capillary damage, as well as the presence or absence of chronic remodeling and rimmed vacuoles. Immunohistochemical staining for class I major histocompatibility complex proteins (MHCI), complement C5b9 (membrane attack complex), and labeling for cytochrome $\mathrm{C}$ oxidase activity (COX) are also often helpful.

\section{Dermatomyositis spectrum-myopathies with rash and perifascicular atrophy}

Dermatomyositis is a systemic disease that is characterized by cutaneous as well as skeletal muscle involvement. It is the most common inflammatory myopathy in children and can represent a paraneoplastic condition in adults. Key clinical features that are considered in the European Neuromuscular Consortium classification system include (1) subacute onset, (2) symmetrical, proximal weakness, and (3) characteristic skin rashes such as heliotrope periorbital edema, Gottron's papules, or erythema of sun exposed skin on chest and neck (V-sign) or upper back (shawl sign). On biopsy, the hallmark feature of dermatomyositis is perifascicular atrophy (Fig. 3a). The atrophic fibers often show loss of COX reactivity (Fig. 3b). Other supporting features include class I major histocompatibility complex upregulation in perifascicular myofibers (Fig. 3d), complement C5b-9 deposition in perifascicular endomysial capillaries (Fig. 3e), and endothelial tubuloreticular inclusions demonstrated on ultrastructural studies. Inflammation in dermatomyositis is typically centered around blood vessels in the perimysium and dominated by CD4-positive T-cells as well as CD20-positive B-cells. Different clinical presentations of dermatomyositis are described. These subtypes may be associated with different antibodies and vary, for example, in the probability of being associated with an underlying malignancy (Table 1) [8]. In the European Neuromuscular Consortium system the clinical diagnosis of "definitive" dermatomyositis requires the presence of typical features including subacute onset, symmetrical proximal weakness and the characteristic skin rashes along with perifascicular atrophy on biopsy. A clinical diagnosis of "probable" dermatomyositis is rendered in cases that exhibit all the clinical features and supporting histologic features but lack perifascicular atrophy. In the absence of sufficient clinical information but characteristic pathological findings, a pathological diagnosis such as "inflammatory myopathy with perifascicular atrophy, consistent with dermatomyositis" is appropriate. In the classification by Pestronk, these cases would typically fall into the category of myovasculopathies [12]. In a large cohort study of 460 patients from Japan, $12 \%$ of the patients with inflammatory myopathies had dermatomyositis [19, 20].

\section{Antisynthetase syndrome and muscle biopsies with perifascicular necrosis}

Some muscle biopsies tend to have more perifascicular necrosis than atrophy $[8,12]$. The presence of abundant perifascicular necrosis with small regenerating fibers resembles dermatomyositis at low power (Fig. 3f) [21], although COX reactivity is typically retained (Fig. $3 \mathrm{~g}$ ). There is evidence that these patients are distinct from those with typical dermatomyositis: firstly, they often have an autoantibody in the anti-tRNA synthetase group (Jo-1, PL7, PL-12, OJ, EJ, Zo, Ha, and KS), with anti Jo-1 being the most common by far. Secondly, there are often distinctive systemic manifestations that include interstitial lung disease, a hyperkeratotic rash on the fingers referred to as "mechanic's hands" and, in some patients, arthralgias. Thirdly, these patients are less likely to have an associated malignancy than adults with dermatomyositis. The perimysial connective tissue often shows widespread pathological changes described as fragmentation and increased alkaline phosphatase reactivity (Fig. 3h). Inflammatory cell infiltrates usually appear to extend from the perimysial into the endomysial compartment. These inflammatory cell infiltrates may include T-cells, B-cells and plasma cells, but may also include perimysial macrophages. Sarcolemmal staining for class I major histocompatibility complex (Fig. 3i) and cytoplasmic staining of myofibers for complement C5b-9 (Fig. 3j) may highlight the perimysiumcentered disease involvement and fiber necrosis. Distinction from dermatomyositis is not always possible by 


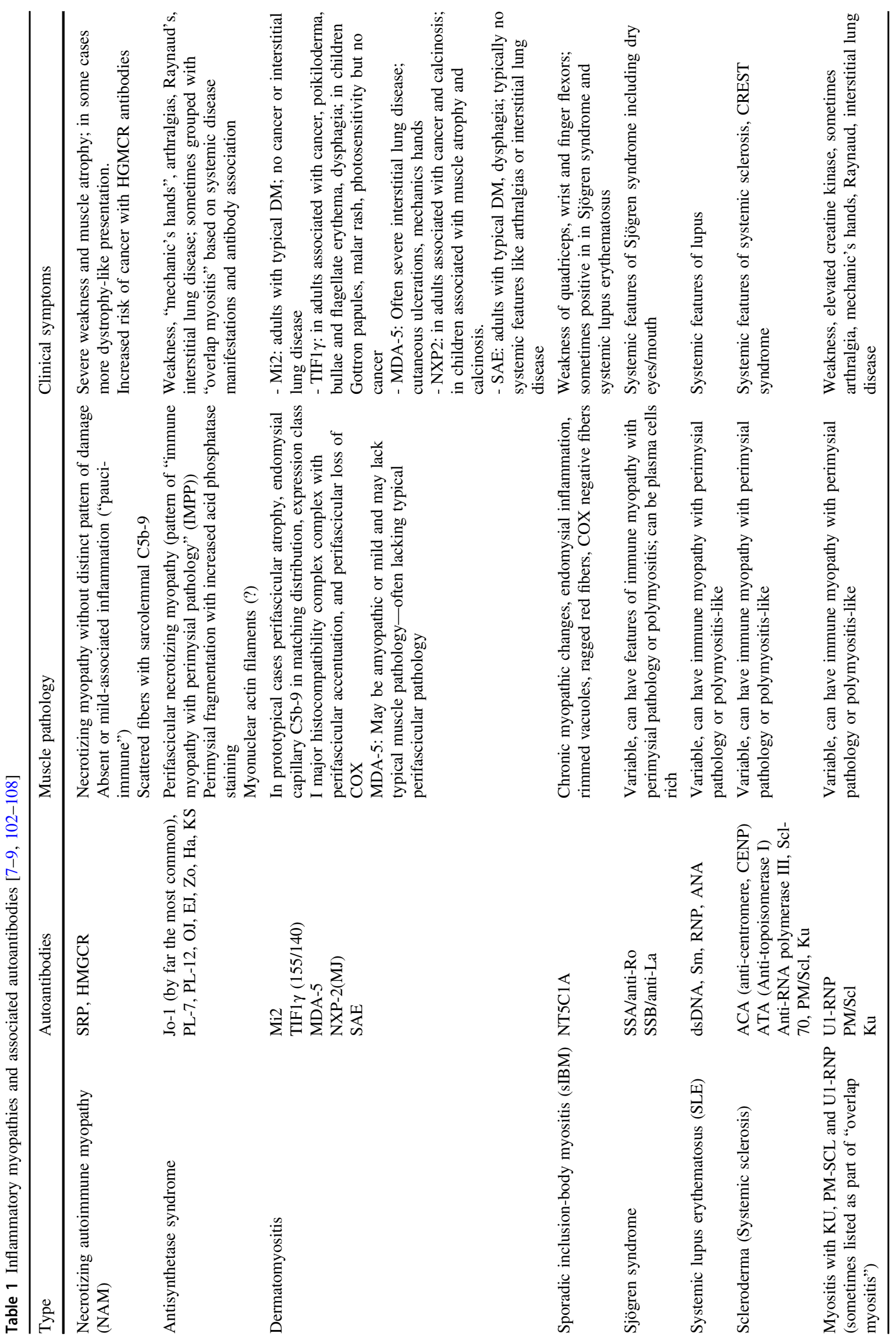



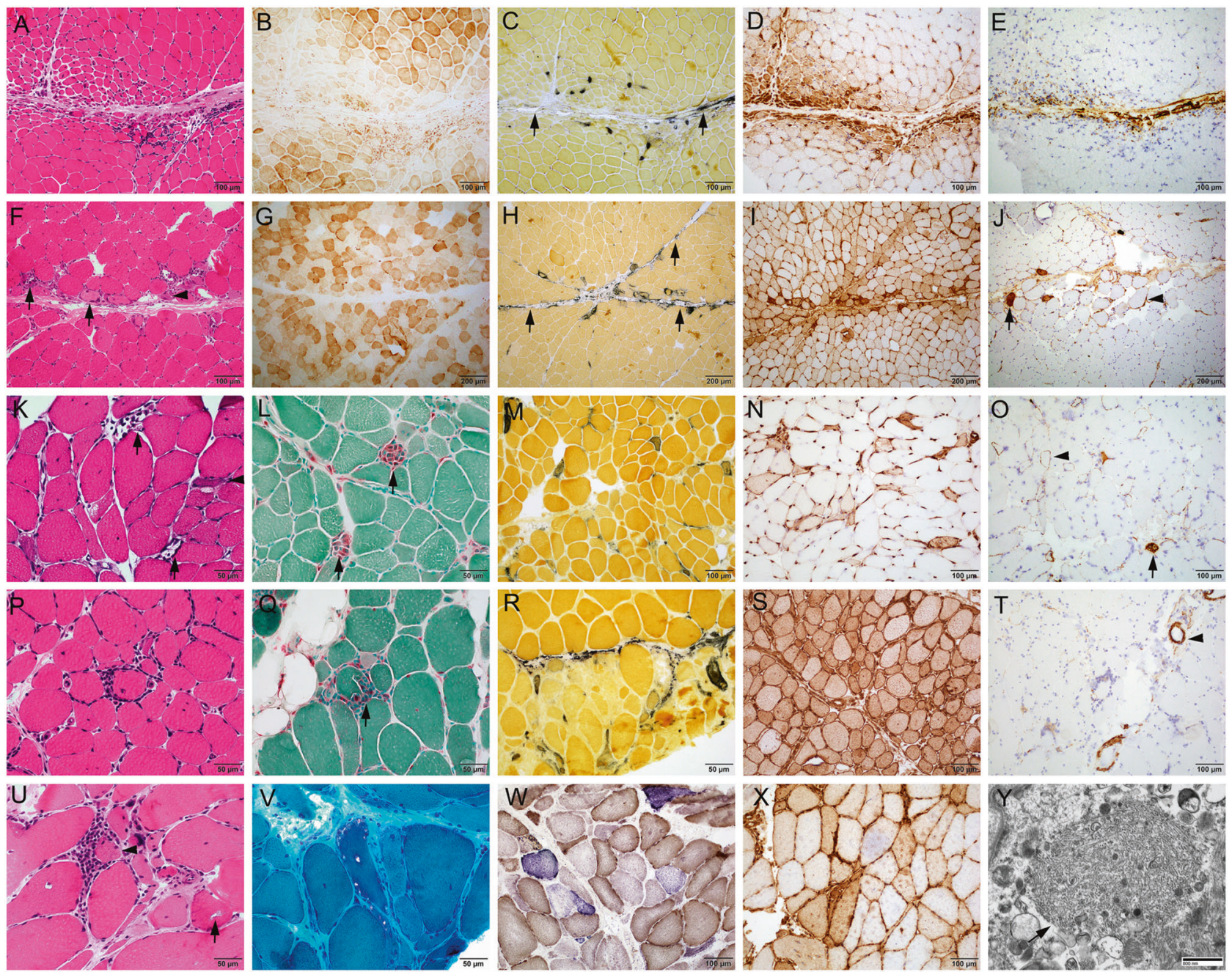

Fig. 3 Inflammatory myopathies and necrotizing myopathies. a-e Dermatomyositis. a H\&E shows prominent perifascicular myofiber atrophy. Necrotic fibers are sparse. b COX stain shows zonal loss of enzyme activity in perifascicular atrophic fibers. c Alkaline phosphatase shows no or focal perimysial connective tissue reactivity (arrows). d Class I major histocompatibility complex immunostain shows high levels of expression in perifascicular fibers. e Complement C5b-9 immunostain shows deposition in perifascicular capillaries. $\mathbf{f}-\mathbf{j}$ Antisynthetase syndrome - Anti Jo-1 myositis. f H\&E shows many necrotic fibers (arrows) and regenerating fibers (arrowheads) in the perifascicular region, but no uniform atrophy. $\mathbf{g}$ There is no zonal loss of COX activity in perifascicular fibers. $\mathbf{h}$ Alkaline phosphatase shows widespread perimysial connective tissue reactivity (arrows). i Class I major histocompatibility complex immunostain shows diffuse myofiber expression, with accentuation in perifascicular fibers. $\mathbf{j}$ Complement C5b-9 highlights necrotic fibers with cytoplasmic staining (arrows) and viable perifascicular myofibers with sarcolemmal staining (arrowheads). k-o Anti-HMGCR necrotizing autoimmune myopathy. k H\&E shows randomly scattered necrotic fibers (arrows) and regenerating fibers (arrowhead). 1 Macrophages in fibers undergoing myophagocytosis (arrows) are positive for acid phosphatase. Alkaline phosphatase (m) and Class I major histocompatibility complex (n)

morphology alone. The diagnosis is supported by detection of serum anti-tRNA synthetase autoantibodies. A recent study suggests that actin aggregates in myocyte nuclei may stains highlight regenerating fibers. o Complement C5b-9 highlights necrotic fibers (arrows) and myofibers with sarcolemmal stain (arrowheads) that are randomly scattered rather than concentrated in perifascicular region. p-t Polymyositis. p Mononuclear inflammatory cells surrounding or invading viable myofiber (arrow) is required for the diagnosis of polymyositis. q Lymphocytes that invade viable myofibers (arrow) are negative for acid phosphatase. $\mathbf{r}$ There may be patchy connective tissue alkaline phosphatase reactivity in PM. s Class I major histocompatibility complex is usually diffusely expressed. $\mathbf{t}$ There is no significant capillary or myofiber complement C5b-9 reactivity. Arterial wall reactivity (arrowhead) is not pathologic and serves as internal staining control. $\mathbf{u}-\mathbf{y}$ Sporadic inclusion-body myositis (sIBM). u Inflammation in IBM is similar to PM, predominantly endomysial and surrounds or invades viable fibers (arrowhead). However, the muscle demonstrates marked chronic changes and vacuoles (arrow). v Red rimmed vacuoles on the modified Gomori trichrome stain. w A COX/SDH double stain highlights frequent COX-deficient fibers. $\mathbf{x}$ Class I major histocompatibility complex shows diffuse or patchy intense expression in myofibers. $\mathbf{y}$ Tubulofilamentous inclusions (arrow) are usually found in vacuoles in sporadic inclusion-body myositis patients

be identified by electron microscopy or immunohistochemistry [22], and can be supportive of the diagnosis. Overall this category corresponds to the "immune 


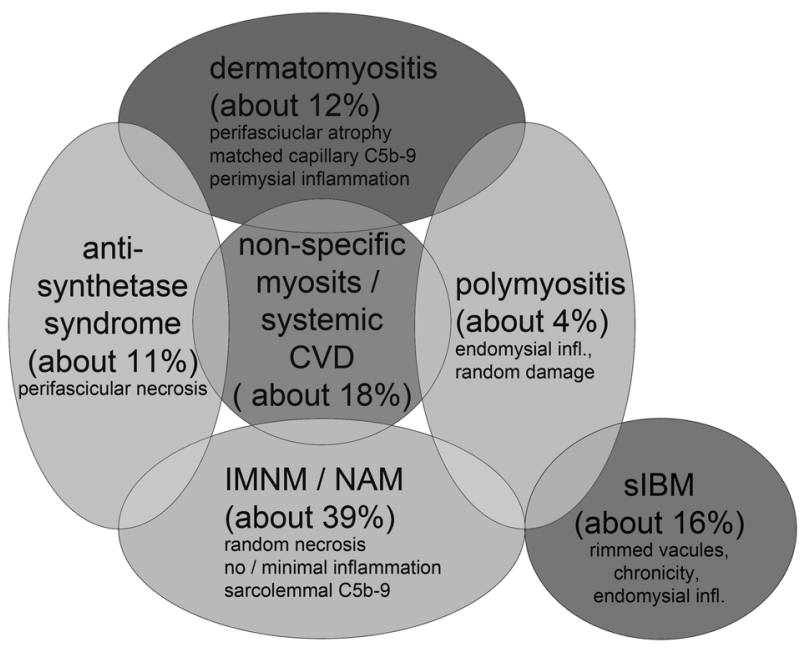

Fig. 4 Venn diagram illustrating the conceptual overlap between different inflammatory myopathies. The classification of inflammatory myopathies has evolved over the last four decades from distinction between polymyositis and dermatomyositis based on the presence of an associated rash to a complex system with overlapping morphologic features. Perifascicular pathology, for example, is found in dermatomyositis as well as in antisynthetase syndrome. The former is usually associated with more atrophy while the latter usually goes along with more necrotizing changes in perifascicular distribution. Polymyositis and sporadic inclusion-body myositis both show endomysial inflammation with CD8-positive T-cells and invasion of normal appearing fibers by inflammatory cells. The most helpful distinguishing feature is the presence of rimmed vacuoles and the associated TDP-43 positivity. Some biopsies lack distinctive morphologic findings. These cases may still ultimately declare themselves as being part of one of the more distinctive categories or may represent muscle involvement by a systemic connective tissue disease. Non-specific myositis associated with systemic connective tissue disease and antisynthetase are together sometimes summarized under the term overlap myositis. The provided percentage numbers represent the distribution of these disease categories in the 460 patients reported by Suzuki et al. [19]. Abbreviations: $C V D$ collagen vascular disease, IMNM immunemediated necrotizing myopathy, $N A M$ necrotizing autoimmune myopathy, sIBM sporadic inclusion-body myositis

myopathies with perimysial pathology (IMPP)" as proposed by Pestronk [12]. In the large Japanese cohort of 460 patients, $11 \%$ of patients with inflammatory myopathies are in the category of antisynthetase syndrome [19]. Some studies utilize the term "overlap myositis" to describe patients with features of inflammatory myopathy that additionally have systemic disease manifestation of autoimmunity and typically are positive for myositis-associated antibodies [9, 15, 23-25]. Patients with antisynthetase syndrome are sometimes grouped together with overlap myositis.

\section{Polymyositis}

On the basis of the classification proposed by Bohan and Peter [10, 11], polymyositis was a common diagnosis. For many years it was to some extend a diagnosis of exclusion rendered in patients with an inflammatory myopathy who did not fulfill the criteria for dermatomyositis or sporadic inclusion-body myositis. With revised classifications, polymyositis has become a much less common diagnosis (Fig. 4). Patients still classified as polymyositis (PM) in the current systems typically present with subacute onset of symmetrical proximal weakness. The serum creatine kinase is often markedly elevated (10-50 times normal). In the European Neuromuscular Consortium system, a diagnosis of definitive polymyositis requires the demonstration of CD8-positive $\mathrm{T}$ lymphocytes surrounding or invading viable myofibers (Fig. 3p, q), associated diffuse class I major histocompatibility complex upregulation in myofibers (Fig. 3s), and the absence of rimmed vacuoles or perifascicular atrophy. These more stringent pathologic criteria have made polymyositis a less common diagnosis $[16,20]$. In the Japanese cohort of 460 patients, only $4 \%$ of the patients had polymyositis [19]. In a subset of polymyositis cases, biopsies are reported to show significant increase in COX-deficient fibers. These cases have been referred to as polymyositis with mitochondrial pathology (PM-mito) or polymyositis with cytochrome oxidase-deficient fibers (PM/ $\mathrm{COX}-$ ). Studies suggest that these patients are less responsive to steroid treatment but may respond to methotrexate $[26,27]$.

\section{Sporadic inclusion-body myositis}

Sporadic inclusion-body myositis (sIBM) is usually discussed with the inflammatory myopathies due to the presence of inflammatory infiltrates. However, unlike the other inflammatory myopathies, it does not appear to have an immune-mediated pathogenesis. The inflammatory cell infiltrates are similar to those of polymyositis, with predominantly T-cells that surround individual myofibers, but the presence of intracellular protein aggregates and the lack of a consistent response to immunosuppression do not support an immune-mediated etiology, and instead have led to comparisons with age-related neurodegenerative diseases. The clinical phenotype of sporadic inclusion-body myositis is quite distinctive with early weakness and atrophy of quadriceps, wrist flexors, finger flexors, and ankle dorsiflexors. Unlike other inflammatory myopathies, muscle weakness is often asymmetrical and progresses slowly over many years. Sporadic inclusion-body myositis is the most common inflammatory myopathy in people over 50 years of age. Serum creatine kinase is normal or mildly elevated. Pathologically, like polymyositis, sporadic inclusion-body myositis often shows CD8+ T-cells surrounding and invading viable myofibers (Fig. 3s). The hallmark of sporadic inclusion-body myositis is the presence of rimmed vacuoles. The rimmed vacuoles contain debris that is red and granular on the modified Gomori trichrome (Fig. 3v) and positive on acid phosphatase. COX negative fibers 
(Fig. 3w) and ragged red fibers are also often encountered in sporadic inclusion-body myositis [28, 29]. Class I major histocompatibility complex is usually diffusely upregulated (Fig. 3x). In contrast to other inflammatory myopathies, sporadic inclusion-body myositis often shows prominent chronic myopathic changes with frequent pyknotic nuclear clumps and hypertrophic fibers that may resemble chronic neurogenic atrophy. On ultrastructural studies, the rimmed vacuoles largely correspond to aggregates of whorled lamellar myelin-like debris, usually located within membrane-bound vacuoles. Although highly characteristic of sporadic inclusion-body myositis, similar autophagic vacuoles may be seen in other disorders associated with abnormal lysosome function. More specific for sporadic inclusion-body myositis is the presence of tubulofilamentous inclusions, which may be present in the vacuoles (Fig. 3y), but may also be present in the nucleus. On special studies the inclusions label for a number of proteins associated with neurodegenerative diseases, including beta amyloid and TDP-43 [30]. The amount of amyloid in the aggregates is often too small to be detectable by Congo red. However, immunostains of p62, LC3, or TDP-43 may highlight these aggregates, even in early sporadic inclusionbody myositis when vacuoles are inconspicuous [31]. In the large cohort of 460 patients from Japan, 16\% of inflammatory myopathies were in the category of sporadic inclusion-body myositis [19].

\section{Necrotizing autoimmune myopathy}

Necrotizing autoimmune myopathy (NAM), also known as immune-mediated necrotizing myopathy (IMNM), results from immune-mediated damage to myocytes. Biopsies from these patients often lack distinctive inflammatory cell infiltrates, but show features of myofiber necrosis. On the basis of the morphologic approach followed in this review, these myopathies are discussed in the next section on biopsies with active myopathic damage without associated inflammation. Pathophysiologically, though, cases of necrotizing autoimmune myopathy belong in the group of inflammatory, or immune-mediated myopathies. In the large cohort of 460 patients from Japan, 38\% of patients with inflammatory myopathies have necrotizing autoimmune myopathy [19].

\section{Myositis without specific diagnostic findings and consideration of the differential diagnosis of inflammatory myopathies}

There are some cases in which a biopsy shows a combination of myopathic damage and inflammation, but lacks sufficient distinctive features to allow a classification into the above specific inflammatory myopathies. These biopsies with inflammation and myopathy fall into three broad categories: (1) cases that may belong to the inflammatory myopathies outlined above but lack sufficient distinctive features on the available biopsy material; (2) cases in which the inflammatory muscle damage represents involvement of skeletal muscle as one component of a systemic connective tissue disease; and (3) cases that may mimic an inflammatory myopathy morphologically but represent an inherited disease process, drug reaction or a local change. In the mentioned large cohort of 460 patients, $18 \%$ of patients with inflammatory myopathies on muscle biopsy were in the non-specific myositis group [19].

(1) Muscle biopsies with inflammation that are equivocal for a definitive classification as a specific inflammatory myopathy.

Despite well-established prototypical features in dermatomyositis, polymyositis, and inclusion-body myositis, none of the morphologic changes are truly pathognomonic. Instead each disease category represents a spectrum that may overlap with other entities for an overall classification system that can be conceptualized as overlapping circles of a Venn diagram (Fig. 4). The following are examples of situation in which biopsy findings may defy an unequivocal classification:

(a) Dermatomyositis and antisynthetase syndrome both exhibit myocyte injury with perifascicular accentuation. Prominent perifascicular necrosis is more supportive of a diagnosis of antisynthetase syndrome while prominent perifascicular atrophy and loss of cytochrome oxidase reaction is more supportive of a diagnosis of dermatomyositis. Some cases of dermatomyositis may, however, show quite prominent necrosis. In such cases, endomysial capillary labeling for complement C5b-9 and tubuloreticular inclusions on electron microscopy were initially considered specific signs for dermatomyositis [14]. Both have, however, more recently also been described in immune-mediated necrotizing myopathy, antisynthetase syndrome, and other autoimmune conditions [7, 20, 21, 32]. Whether myonuclear actin aggregates will be confirmed as reliable marker of antisynthetase syndrome, will have to await further studies [22].

(b) Perifascicular distribution of necrosis or atrophy may be variable and is sometimes absent in biopsies of patients with dermatomyositis and antisynthetase syndrome. In some cases of dermatomyositis, the perifascicular distribution of sarcolemmal class I major histocompatibility complex expression may be the only abnormal finding on muscle biopsy. The lack of unequivocal perifascicular myofiber muscle damage or atrophy does not exclude these diagnoses.

(c) Muscle biopsies in sporadic inclusion-body myositis 
muscle may have sparse vacuoles and minimal chronic changes in the early stages of the disease. The distinction between polymyositis, polymyositis with mitochondrial pathology, and sporadic inclusionbody myositis may, therefore, be difficult [33]. Immunostaining of p62 and TDP-43 may highlight protein aggregates in sporadic inclusion-body myositis and help separate these two entities [31, 34, 35].

(d) Rimmed vacuoles are often described as the hallmark of sporadic inclusion-body myositis. These inclusions are, however, also seen in hereditary inclusion-body myositis (hIBM) as well as in some muscular dystrophies [36-39]. On occasion, rimmed vacuoles may even be present in the context of neurogenic atrophy [40]. Furthermore, a number of other inclusions may at times exhibit morphologic features that overlap with those of rimmed vacuoles including mitochondrial aggregates, inclusions of chloroquine or colchicine toxicity, and aggregates of storage diseases. Sometimes the diffuse expression of class I major histocompatibility complex, endomysial lymphocytic inflammation, and the appropriate clinical history can be very helpful in differentiating sporadic inclusion-body myositis from hereditary forms.

(2) Muscle involvement in systemic connective tissue diseases.

Skeletal muscle involvement is not just a feature of the described "primary" inflammatory myopathies but can instead also be seen in many systemic connective tissue diseases, including systemic lupus erythematosus, rheumatoid arthritis, scleroderma, Sjögren syndrome, and sarcoidosis [23, 24, 41-44]. Sarcoid myopathy typically shows non-caseating granulomatous inflammation, but the others may variably mimic other inflammatory myopathies like polymyositis, dermatomyositis, necrotizing autoimmune myopathy or antisynthetase syndrome on biopsy. The recognition of typical clinical features of a systemic connective tissue disease as well as autoimmune associated antibodies summarized in Table 1 can offer helpful clues to the diagnosis. The term "overlap myositis" has been used to describe patients with an overlap presentation between inflammatory myopathy and systemic connective tissue disease. These patients exhibit features of systemic connective tissue diseases beyond muscle involvement and are typically positive for myositis-associated antibodies [9, 15, 23-25].

(3) Inflammatory changes in other diseases.

Inflammatory infiltrates may sometimes be encountered with inherited diseases like muscular dystrophies. In that context, they represent a secondary process and may cause diagnostic confusion. Inflammatory changes may also be seen in various other settings. These include patients with checkpoint inhibitors [45, 46], localized or focal myositis as a poorly understood inflammatory process that presents as localized inflammatory pseudotumor [47], infections, or vasculitis (Fig. 2).

\section{Section II: Active myopathic damage without associated inflammation}

Some biopsies exhibit active myopathic damage with ongoing myofiber necrosis and regeneration, but lack inflammatory infiltrates or features of chronicity. Active myofiber breakdown leads to release of muscle enzymes and may result in the clinical presentation of rhabdomyolysis or myoglobinuria. Severe rhabdomyolysis is, in many cases, the result of a monophasic injury such as trauma with crush injury, exertion, hyperthermia, infections, drugs, or toxins. The monophasic nature of the injury is characteristically evident in the muscle biopsy specimen by abundant damaged fibers all at similar stage of necrosis or regeneration. Muscle biopsies are usually not performed in these settings, especially if the cause is clinically apparent such as rhabdomyolysis during flu or toxin exposure. Scenarios that may require a muscle biopsy are persistently elevated serum creatine kinase levels, acute or subacute onset of progressive weakness, or repeated episodes of rhabdomyolysis. The main diagnostic considerations for biopsies with the pattern of only active myopathic damage not associated with inflammatory cell infiltrates include necrotizing autoimmune myopathies, exogenous myotoxins or drugs, and metabolic myopathies.

\section{Necrotizing autoimmune myopathy}

Necrotizing autoimmune myopathy (NAM; also referred to as immune-mediated necrotizing myopathy IMNM) often presents acutely, with very high creatine kinase levels and severe generalized weakness. The clinical spectrum of this disease is, however, still evolving and cases with a more, chronic dystrophy-like presentation are also described [48, 49]. Most patients with necrotizing autoimmune myopathy have either anti-SRP or anti-HMGCR antibodies $(75 \%$ in one study (20)). The clinical features associated with antiHMGCR and anti-SRP antibodies largely overlap, but patients with anti-SRP antibodies more often show muscle atrophy and weakness of neck and respiratory muscles as well as pharyngeal muscles creating a pattern of weakness that may at times mimic facioscapulohumeral muscular dystrophy (FSHD) discussed in Section III [20]. Extramuscular manifestations such as interstitial lung disease and cardiac involvement are relatively uncommon, but if present more likely associated with anti-SRP antibodies. AntiHMGCR antibodies were initially identified in patients with 
prolonged weakness after statin usage that persisted after discontinuation of the drug $[50,51]$. But only some of the patients with anti-HMGCR antibody have had prior statin exposure rendering the term "statin-induced autoantibodies" inaccurate. Pathologically, the muscle shows necrotizing myopathy with scattered necrotic fibers at different stages of regeneration, but only mild or no overt inflammatory cell infiltrates (Fig. 3k-o). Necrotizing autoimmune myopathy with anti-SRP antibody is often severe, with rapid development of interstitial fibrosis [12]. Class I major histocompatibility complex expression is usually not as prominent as in other inflammatory myopathies and is often patchy or limited to individually scattered myofibers (Fig. 3n). Complement C5b-9 deposition on the sarcolemma of scattered viable myofibers (Fig. 3o) may be seen in a significant subset of patients.

\section{Myotoxic myopathy}

Toxic myopathy may result from exposure to alcohol, opiates and other street drugs, statins, and chloroquine/ hydroxycloroquine [52]. In most of these instances, biopsies exhibit acute myopathic features without a background of inflammatory changes. If exposure to the inciting agent is terminated, the symptoms usually resolve and no further treatment is required. Statin use may result in two distinct patterns of muscle injury: direct statin toxicity and necrotizing autoimmune myopathy with HMGCR antibodies [53, 54]. With direct statin toxicity, patients typically improve after discontinuation of the drug, while symptoms due to necrotizing autoimmune myopathy typically persist after discontinuation of the medication and require immunosuppressive medications. Specific diagnostic features may be identifiable in some medication-induced myopathies. Chloroquine and colchicine are associated with vacuolar myopathy described in detail below (Section IV). Zidovudine, an antiretroviral drug in HAART treatment for AIDS patients, inhibits mitochondrial DNA polymerase and may result in mitochondrial myopathy with ragged red fibers and COX-deficient fibers as discussed in Section IV [55]. Alcohol-related rhabdomyolysis is associated with binge drinking episodes while chronic alcoholism has been linked to type II atrophy $[56,57]$.

\section{Metabolic myopathy}

Metabolic myopathies may be divided into two groups based on the pattern of clinical presentation. Many result in episodic symptoms that are often precipitated by exercise, fasting, or infection while others cause progressive muscle damage more akin to a muscular dystrophy [58]. In the former, it is recommended that the biopsy be performed at least one month after a rhabdomyolysis episode, because extensive fiber necrosis and edema during the acute phase may obscure the underlining pathology and may preclude biochemical comparisons to normal muscle in metabolic etiologies. It is important to biopsy a moderately weak muscle to increase the diagnostic yield. The metabolic diseases are discussed in more detail in Section IV because they often show formation of cytoplasmic vacuoles or inclusions.

\section{Differential diagnosis}

An etiologic diagnosis is often impossible based solely on the morphologic changes of active myopathic damage. Many of these cases represent necrotizing autoimmune myopathy. Class I major histocompatibility complex and complement C5b-9 immunostains may be helpful since appropriate patterns of positive staining would favor this diagnosis. Serological testing for myositis specific antibodies (SRP, HMGCR, Jo-1, and other antisynthetase syndrome-associated antibodies) can provide additional helpful information. Negative results could favor toxic or metabolic disorders, but do not exclude necrotizing autoimmune myopathy. In some cases, a muscle biopsy from a patient that may otherwise fit into categories like polymyositis or myositis without specific structural findings may lack diagnostic inflammatory cell infiltrates due sampling artifact or prior immune modulating therapy. These inflammatory myopathies are, therefore, not entirely excluded based on the lack of inflammation.

\section{Section III: Chronic myopathic changes}

The combination of myopathic changes together with features of disease chronicity in the form of endomysial fibrosis and fatty replacement implies a longer-standing smoldering disease process. These changes are reflective of the time course of the disease process rather than any particular etiology. The extent of active necrosis varies depending on the underlying disease process and is often related to the tempo of clinical deterioration. Chronic myopathic changes can be encountered in muscular dystrophies, some congenital myopathies, some metabolic myopathies and even inflammatory myopathies-most often inclusion-body myositis (see Section I). The patient's age, family history, the duration of symptoms, the distribution of weakness, and the involvement of other organ systems can all provide useful clues in developing a differential diagnosis.

Muscular dystrophies and congenital myopathies represent two broad groups of inherited myopathies. In principal, the prototypical muscular dystrophy is a disease that affects patients who appear normal at birth but subsequently 
develop progressive muscle weakness, with myofiber necrosis also reflected in elevated serum creatine kinase levels. Duchenne muscular dystrophy represents the most common and a typical example. Congenital myopathies on the other hand often result in weakness, hypotonia, or muscle contractions that are apparent in early infancy [59, 60]. Severe cases can lead to early death but many forms result in a more static course, depending on the causative mutation. Muscle damage is typically not as relentlessly progressive as in a muscular dystrophy and is usually associated with distinct morphologic features like nemaline rods or central cores. Congenital muscular dystrophies and myofibrillar myopathies are two additional categories of inherited muscle diseases besides dystrophies and congenital myopathies [61, 62]. All of these broad categories should be understood as reflective of general concepts in the approach to the dauntingly diverse group of genetic muscle diseases. The borders between these disease groups are not as sharp as this outline may imply.

\section{Muscular dystrophies}

The muscular dystrophies represent a challenging group of diseases for the pathologist examining muscle biopsies because they typically lack distinctive changes beyond the features of chronic muscle damage (Figs. 2, 5). For a subgroup, immunohistochemical stains for sarcolemmal proteins can serve as markers for the underlying mutations when the mutation prevents normal expression of proteins like dystrophin and dysferlin. But in many cases, the muscle biopsy does not provide the genetic classification. In these cases, the genetic diagnosis is only rendered after integration of the clinical history, pattern of weakness and associated other systemic features with results of genetic testing (Fig. 5).

Muscular dystrophies are sometimes broadly categorized as X-linked dystrophinopathies, autosomal limb girdle muscular dystrophies, myotonic dystrophy, and other more rare forms that are recognized by their striking pattern of muscle involvement often limited to certain anatomic sites, like facioscapulohumeral muscular dystrophy (FSHD), oculopharyngeal muscular dystrophy, and the elbow contractures commonly seen in X-linked Emery-Dreifuss muscular dystrophy [63-65]. The role of muscle biopsy in the workup of these disorders has changed markedly over the last several years. This change has been driven primarily by the rapid development of genetic tests for these diseases. Detailed analysis of skeletal magnetic resonance imaging may also provide important diagnostic clues because many dystrophies exhibit a strikingly distinctive pattern of muscle involvement that arises out of differences in which muscles are affected by the disease [66, 67]. At the present time, the diagnosis of Duchenne no longer requires a biopsy, but is
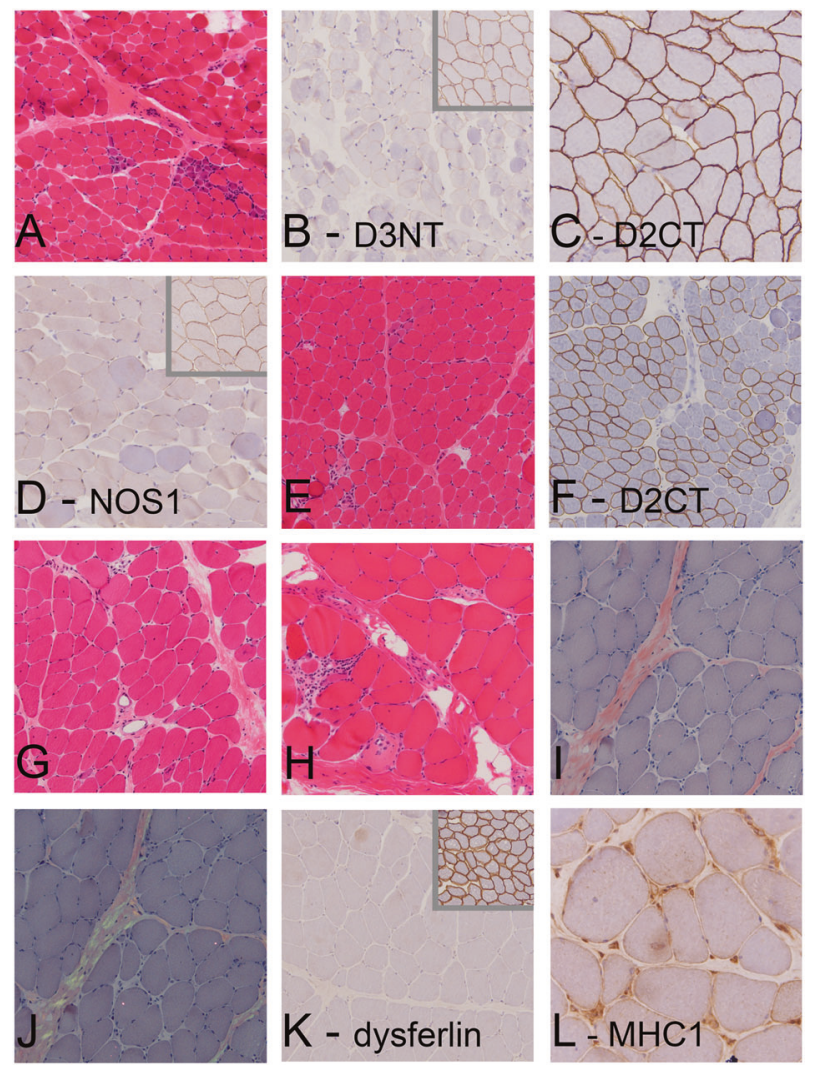

Fig. 5 Muscular dystrophies. a-d Dystrophinopathy with unusual dystrophin mutation missed on initial testing. The patient was a young boy who was initially found to have elevated liver function tests, and subsequently found to have elevated creatine kinase levels. Initial genetic testing for Duchenne muscular dystrophy was negative. The biopsy still suggests a dystrophin mutation with a distinctly patchy small clusters of either degenerating or regenerating myofibers, $\mathbf{b}$ loss of staining for the $\mathrm{N}$-terminus of dystrophyin (insert shows normal control) but preserved reactivity for the C-terminus of dystrophin (c) and $\mathbf{d}$ loss of normal membranous labeling for NOS-1 (insert shows normal control). Further testing found a mutation at an intron-exon boundary of dystrophin. e, f Symptomatic carrier of dystrophin mutation. The patient was a 5-year-old girl with exercise-induced weakness and muscle cramping. A biopsy showed myopathic changes on H\&E stained sections with focal degenerating and regenerating myofibers (e). The special stains showed patchy loss of all the tested epitopes of dystrophin, a common finding in symptomatic carriers (staining for the C-terminus is shown, f). g Limb girdle muscular dystrophy (LGMD) with titin mutation. The patient's biopsy shows mild myopathic changes with focal myofiber necrosis and regeneration (g). Immunohistochemical staining for dystrophyassociated proteins was performed because of a long-standing history of muscle weakness but was within normal limits. Genetic testing identified two titin variants that are predicted to be pathogenic suggesting a diagnosis of titin deficient limb girdle muscular dystrophy (LGMD2J), and the biopsy confirms a dystrophic pattern of myocyte involvement. h-l Limb girdle muscular dystrophy with dysferlin mutation (LGMD 2B). The biopsy of this young adult patient with proximal muscle weakness shows myopathic changes with myofiber degeneration and regeneration (h). A Congo red stain is positive for congophilic material with green birefringence $(\mathbf{i}, \mathbf{j})$. On immunohistochemical staining expression of dysferlin is absent (k; insert shows positive control). Focal increased sarcolemmal staining for class I major histocompatibility complex is seen (l) and focal lymphocytes are present 
rendered by the identification of a known causative mutation. Variably comprehensive genetic panels offered by diagnostic laboratories cover many other dystrophies. The pathologist, therefore, rarely encounters biopsies from prototypical cases of dystrophies these days. Instead, the question of an underlying muscular dystrophy usually arises in the setting of a patient with unusual clinical features or in a patient that presents later in life with clinical features that mimic acquired myopathies. In some instances, a biopsy may be performed because genetic testing identifies a variant of uncertain significance in a dystrophy-associated gene with the hope of providing additional diagnostic conformation. In other cases, the results of the initial genetic testing may not match the clinical diagnosis (Fig. 5a-d).

\section{Dystrophinopathies (X-linked dystrophies with dystrophin mutations including Duchenne and Becker)}

Duchenne muscular dystrophy is the prototypical example of a muscular dystrophy and represents the most common type of the X-linked dystrophinopathies-diseases caused by mutations in the dystrophin gene. Other clinical manifestations of dystrophinopathies include the milder variant known as Becker muscular dystrophy, as well as rare symptomatic carries, rare patients with predominantly cardiac phenotype, and rare patients in whom the disease presents as exercise-induced rhabdomyolysis. In general, there is a genotype-phenotype correlation: mutations that prevent any dystrophin synthesis by introducing an earlystop codon or frame shift typically cause Duchenne muscular dystrophy; mutations that are less disruptive may lead to expression of a shortened or only partly functional form of the protein. These latter patients typically have a milder clinical course that may, for example, follow that of Becker muscular dystrophy. Dystrophin is part of the dystrophin-glycoprotein complex that provides a link between the cytoskeleton inside the cell and the basement membrane outside the myofibers. Disruption of this protein complex is thought to lead to focal disruption of the sarcolemma during contraction that allows calcium entry and, ultimately, cell death. Continuous muscle damage may outpace the muscle's regenerative capacity, leading to the progressive loss of muscle mass and increasing chronic remodeling that manifests as the relentlessly progressive course of muscular dystrophies (Fig. 2). Typical cases of Duchenne are easily confirmed on immunohistochemical studies of biopsies because antibodies to different domains of this large $400 \mathrm{kD}$ protein all show loss of expression. In most practices, biopsies for Duchenne have become very uncommon due to the transition in the diagnostic approach towards genetic testing. Biopsies from patients with unusual mutations or other forms of dystrophinopathies are, however, still encountered (Fig. 5a-f). The workup of these samples by immunohistochemistry can be challenging because only some domains may be disrupted resulting in loss of staining with only selected antibodies. Addressing the question of a dystrophinopathy on a muscle biopsy, therefore, requires the use of a panel of antibodies recognizing different parts of the protein and is often complemented by staining for nitric oxide synthase 1 (NOS-1) $[68,69]$. NOS- 1 is anchored to its subsarcolemmal location by dystrophin. Its normal sarcolemmal staining pattern is, therefore, lost in cases of dystrophinopathy. It is important to establish an accurate diagnosis to guide further management. Follow-up by a cardiologist represents an important element of care for patients with dystrophinopathies due to the common cardiac involvement in dystrophinopathies. In addition, there have been some promising advances towards treatment of Duchenne muscular dystrophy including exon skipping or early-stop codon read through that require an exact description of the underlying genetic defect [70].

\section{Limb girdle muscular dystrophies}

The limb girdle muscular dystrophies (LGMDs) represent a large group of over 30 different diseases with autosomal dominant or recessive inheritance pattern [71]. As the name implies, these diseases typically share a pattern of weakness with accentuated involvement of proximal muscle groups. Historically, they were defined by an autosomal inheritance pattern, a pattern of proximal muscle involvement of the limbs, high creatine kinase levels, and prominent dystrophic myopathy but also the lack of typical features of myotonic dystrophy. Today the limb girdle muscular dystrophies are recognized as diverse group of genetically defined diseases. The individual genes are often linked to other allelic disease manifestations [71]. Patients with dysferlin mutation, for example, sometimes develop a phenotype of distal lower extremity weakness instead of limb girdle muscular dystrophy, and some patients with FKRP mutations sometimes present as congenital muscular dystrophy rather than limb girdle muscular dystrophy. The incidence of different limb girdle muscular dystrophies varies by geographic regions but overall calpainopathies, dysferlin deficiency, sarcoglycanopathies, and dystroglycanopathies are the most common forms [71-74]. Most limb girdle muscular dystrophies show chronic active myopathy on biopsy, often with necrosis and fibrosis (Fig. 5). Dysferlinopathy (LGMD 2B) is detailed below as an example and because it demonstrates some unique diagnostic features.

Dysferlin is a transmembrane protein that is expressed in skeletal muscle and thought to have a role in regulating vesicle trafficking and membrane resealing after injury. Mutations in dysferlin are the genetic cause for limb girdle muscular dystrophy 2B (OMIM 253601) but are also linked 
to other more rare presentation including Miyoshi myopathy (OMIM 254130), distal myopathy with anterior tibial onset (OMIM 606768) and exercise-induced rhabdomyolysis. Patients with limb girdle muscular dystrophy $2 \mathrm{~B}$ may have variable onset of symptoms but are often adolescents or young adults. Creatine kinase levels are markedly elevated in most patients and some are initially thought to suffer from an inflammatory myopathy. The distinction of limb girdle muscular dystrophy $2 \mathrm{~B}$ from acquired inflammatory myopathies may also be challenging for the pathologist because features of chronicity are often subtle early in the disease course, and because inflammatory infiltrates and class I major histocompatibility complex upregulation are not infrequent findings. The basic histomorphology may not resolve this differential diagnosis but immunohistochemical staining for dysferlin can help to identify the loss of normal protein expression as a clue to the underlying biologic process (Fig. 5h-1). Limb girdle muscular dystrophy $2 \mathrm{~B}$ and facioscapulohumeral dystrophy are the two types of dystrophies that most frequently show mononuclear inflammatory cell infiltrates as a red herring that can lead to the erroneous diagnosis of an inflammatory process but the same phenomenon is also described in other dystrophies [75, 76]. A second unusual morphologic feature of the myopathy associated with dysferlin deficiency is the presence of amyloid deposition in some cases [77].

\section{Congenital myopathies}

The group of congenital myopathies, sometimes also known as the "structural myopathies", is, to some extent, more satisfying to the histopathologist because they are defined by morphologic changes, such as nemaline rods or central cores [59, 78]. The basic diagnosis of congenital myopathies is, therefore, relatively straightforward for those patients that present in a typical fashion with weakness and hypotonia in infancy or early childhood. Some patients, however, do not come to attention until late childhood or early adulthood, despite the name "congenital". In these cases, the diagnosis may be more challenging. A diagnosis of congenital myopathy is typically not a major consideration in these older patients and the pathologic diagnosis may, therefore, be an unexpected finding [79]. In these cases, the biopsy typically shows type I predominance and selective type I atrophy/ hypotrophy. The pathologic changes may also include features of chronic remodeling with fibrosis and fatty replacement. The diagnostic histologic findings may sometimes be obvious but are in other cases only found after examination of special stains and careful study of the biopsy. In addition, the genetic basis cannot be determined based on histomorphology. Central core disease, for example, may result from mutations in a number of genes including RYRl, NEB, SEPN1, ACTA1, and KBTBD13. There is some morphologic overlap between targetoid formations seen in the context of denervation, central cores and mini-cores. Nicotinamide adenine dinucleotide tetrazolium reductase (NADH-TR) reaction, succinate dehydrogenase $(\mathrm{SDH})$ reaction, $\mathrm{COX}$ reaction as well as ultrastructural studies can help to define core-type inclusions. Central cores are clearly demarcated mitochondria free zones (Fig. 6a) that retain ATPase reactivity (Fig. 6b). The background muscle fibers show type I predominance. The cores are usually single and centrally located. On electron microscopy, unstructured cores show large areas of Z-band streaming that extend longitudinally parallel to the long axis of the myofibers (Fig. 6c) while structured cores are well demarcated mitochondria-free zones with only subtle Z-band irregularity (Fig. 6d, arrows). Minicores may be quite irregular and subtle on light microscopy (Fig. 6e arrow) in which case electron microscopy can help to identify multiple small areas of Z-band irregularities, often limited to just the length of two or three sarcomeres (Fig. $6 f$ arrows). Multiple separate mini-cores may be found within one fiber. Well-formed target fibers contain a clear central zone, a dark intermediate zone and a pale peripheral zone on NADH-TR staining (Fig. 6g). In practice, however, "targetoid" changes are more common and very similar to central cores on light microscopy and ultrastructure examination. The distinction often relies on the overall context. The diagnosis of central core disease or disease with coretype inclusions may be confirmed by genetic testing. In lateonset cases, the phenotype may be dominated by axial weakness [79, 80]. A diagnosis of central core disease with RYR1 mutation has additional potential implications for the patient because of the association of some RYRI mutations with malignant hyperthermia.

\section{Sporadic late-onset nemaline myopathy}

An interesting twist on the story of muscle biopsies with features of congenital myopathies, or mimicking congenital myopathies, is seen in those patients that are now classified by some as sporadic late-onset nemaline myopathy (SLONM) [81, 82]. The biopsies of affected patients show features of nemaline myopathy with chronic atrophic changes and clusters of nemaline rods that are often best seen on the modified Gomori trichrome stain (Fig. 6h) and confirmed by electron microscopy (Fig. 6i). These nemaline rods are easily missed on initial inspection of routine histologic slides. It is, therefore, good practice to specifically look for abnormally coarse granular inclusions on the modified Gomori trichrome stain when examining a biopsy of an elderly person with otherwise non-specific atrophic or mild chronic myopathic changes. The presentation in patients with sporadic late-onset nemaline myopathy is, as implied by the name, thought to be sporadic. Most patients are in their fifties or sixties and present with slowly 

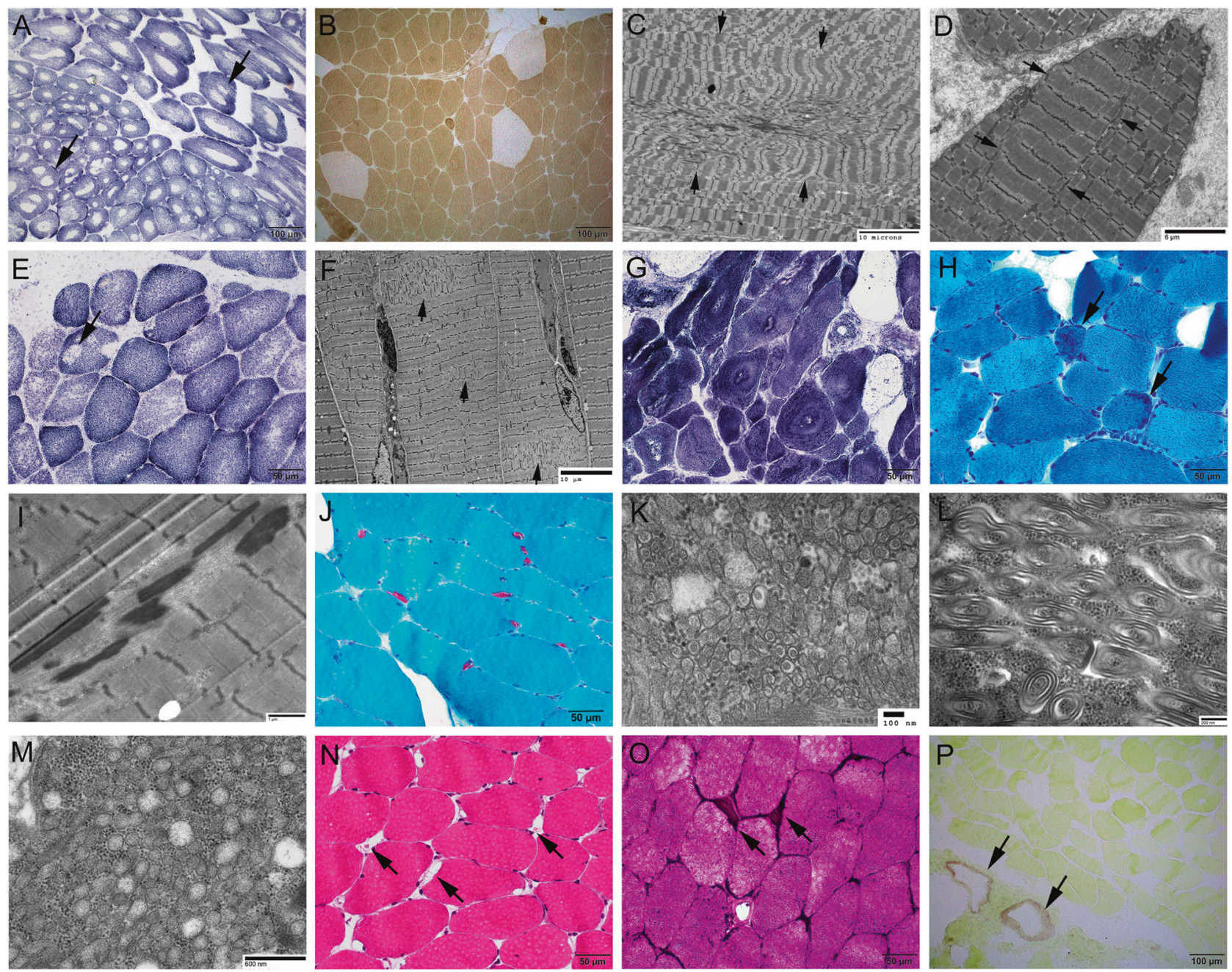

Fig. 6 Cores, targets, inclusions, and vacuoles. a-c Central core disease in a 10-year-old boy with a RYRl mutation. a SDH highlights numerous central cores; a few fibers contain multiple cores (arrows). b ATPase $\mathrm{pH} 4.3$ show type I fibers (dark) predominance and atrophy. Type II fibers (light) are hypertrophied. c Electron micrograph showing unstructured cores. Arrows delineate boundary of the mitochondria free zone. The center shows Z-band streaming. d Structured core from a 2-year-old female with central core disease, malignant hyperthermia, and RYRI mutation. Arrows delineate mitochondria free zone. e, f Multi-minicore disease in a 2-year-old female with $M Y H 7$ mutation. e SDH. f Electron microscopy. g Target fibers on NADH stain in an adult patient with amyotrophic lateral sclerosis. h, i

progressive weakness. In some patients, the disease is accompanied by a monoclonal gammopathy, human immunodeficiency virus (HIV) infection, or another immune disorder and some patients improve if the underlying disease is treated successfully.

\section{Differential diagnosis}

In some instances, a muscular dystrophy is initially mistaken for a different disease process because the biopsy exhibits features not typically seen in a dystrophy, such as

Sporadic late-onset nemaline myopathy. Gomori trichrome (h) and electron microscopy (i) images show nemaline myopathy in a 61-yearold man with lupus. $\mathbf{j}-\mathbf{m}$ Tubular aggregate myopathy. $\mathbf{j}$ Gomori trichrome. $\mathbf{k}$ EM: tubular aggregates. I Electron microscopy: cylindrical spirals. m Electron microscopy: vesicular membranous aggregate. (k, $\mathbf{l}, \mathbf{m}$ are from three separate patients). $\mathbf{n}-\mathbf{p}$ Glycogen storage disease type V (McArdle disease) from a 14-year-old female with exerciseinduced cramp and rhabdomyolysis. n H\&E shows subsarcolemmal vacuoles (arrows). o Non-aqueous PAS without diastase showing glycogen accumulation (arrows). p Myophosphorylase enzyme histochemical stain shows no enzyme activity in myofibers but retained expression in vessels (arrows)

mononuclear inflammatory cell infiltrates. The pitfall of erroneously labeling a dystrophy as inflammatory myopathy has already been discussed [75, 76]. On the other hand, some patients that are initially classified as having a muscular dystrophy are only later discovered to have a different disease. For example, some patients with late-onset Pompe disease may initially be thought to have limb girdle muscular dystrophy [83], and some patients with immunemediated necrotizing myopathy may have an initial clinical presentation that mimics muscular dystrophy [19, 48, 49]. Sporadic inclusion-body myositis (discussed in Section I) 
also shows chronic myopathic changes on muscle biopsy similar to those seen in dystrophies. The presence of inflammation, and rimmed vacuoles as well as the patient age are typically thought to set sporadic inclusion-body myositis aside from dystrophies. In this context it is, however, important to keep in mind that rimmed vacuoles are not pathognomonic for (sporadic) inclusion-body myositis but also found in rare hereditary diseases, including hereditary inclusion-body myopathy as well as other rare chronic myopathies [36-39]. The importance of considering other diagnosis, therefore, increases if histomorphology and clinical context diverge from what would typically be expected in sporadic inclusion-body myositis.

\section{Section IV: Myopathies with distinctive inclusions or vacuoles}

Normal myofibers are characterized histologically by a uniform fine internal reticular pattern that may be see on H\&E stained sections and is highlighted by the modified Gomori trichrome stain. This pattern is the result of the uniform regular arrangement of myofibrillar bundles surrounded by cytoplasm, including glycogen and membranous organelles, such as sarcoplasmic reticulum and mitochondria. Disruption of this normal internal architecture, aggregation of proteins, or accumulation metabolites like glycogen may result in vacuoles or inclusions that in turn provide clues to the underlying diagnosis.

\section{Mitochondrial aggregates and mitochondrial myopathies}

Mitochondrial diseases are the great mimicker in the world of neuromuscular diseases: They may present at any age and may cause peripheral neuropathy, progressive myopathy or acute exercise-induced rhabdomyolysis. In addition, they can be associated with various systemic manifestations ranging from endocrinopathy to cardiomyopathy to seizures or gastrointestinal problems. The genetics of mitochondrial diseases are complex with nuclear encoded defects inherited as Mendelian traits and mitochondrial DNA variants that show a maternal pattern of inheritance [84]. A muscle biopsy from an affected patient may exhibit obvious features of myopathic damage or may only exhibit mild fiber atrophy. However, three morphologic changes are important to recognize as suggestive of a diagnosis of a mitochondrial disease, (1) ragged red fibers (RRF), (2) COX negative fibers, and (3) strongly SDH reactive blood vessels:

(1) Ragged red fibers result from the aggregation of mitochondria within the cytoplasm. Typically these are found in subsarcolemmal distribution where they result in a rind-like thickening that is often already appreciable on the H\&E section and better visualized on the modified Gomori trichrome stain. The reddish color of the granular subsarcolemmal mitochondrial aggregates on Gomori trichrome stain is the origin of the name, ragged red fibers. Staining for SDH also highlights the subsarcolemmal mitochondrial aggregates and may aid in distinguishing them from other inclusions that may at times share similar morphology on the trichrome stained sections. Ultrastructural studies confirm the presence of mitochondrial aggregates and may further identify morphologically abnormal mitochondria, such as those with intramitochondrial paracrystalline inclusions ("parking lot structures") or concentric membranous arrangement of cristae ("phonograph records").

(2) COX-negative fibers may appear normal on $\mathrm{H} \& \mathrm{E}$ sections, but the enzyme histochemical staining confirms the absence of enzyme activity. The presence of scattered myocytes that do not express any cytochrome oxidase activity is strong supporting evidence of a mitochondrial myopathy.

(3) Cases of mitochondrial myopathy, encephalopathy, lactic acidosis and stroke-like episodes (MELAS) have been reported to show perimysial muscular blood vessels with markedly increased SDH reactivity [85].

The presence of ragged red fibers or COX negative fibers may suggest a diagnosis of mitochondrial disease in the appropriate clinical context [84]. Ragged red fibers and COX negative fibers can, however, also be part of normal, age-related changes. There is no widely accepted threshold for how many ragged red fibers or COX negative should be considered substantially increased. Bernier et al. suggested a cutoff of $>2 \%$ ragged red fibers for any age, $>2 \%$ COXnegative fibers in patients between age 30-50 years, and $>5 \%$ COX-negative fibers in patients over 50 years as major criteria for mitochondria myopathy [86]. Any ragged red fibers in patients under 30 year of age, 1-2\% ragged red fibers in patients between 30 and 50 years, and $>2 \%$ fibers with subsarcolemmal mitochondria accumulation in children under 16 years would be considered minor criteria. Several other notable exceptions are listed below. The decision regarding further biochemical or genetic testing depends on the details of the clinical context.

1. Ragged red fibers and COX negative fibers may be seen in large numbers in Zidovudine (AZT) toxicity, sporadic inclusion-body myositis and some cases of polymyositis $[28,29,55,87,88]$. An important clinical clue to separate primary mitochondrial 
myopathy from sporadic inclusion-body myositis can be external ocular muscle weakness (ophthalmoplegia), which is a common features in patients with mitochondrial myopathy but not usually found in sporadic inclusion-body myositis patients.

2. External ocular muscles and axial muscles such as paraspinal and scalene muscles may have a high number of COX-negative fibers without association with a mitochondrial disorder. The consensus report from the mitochondrial medicine society recommends using vastus lateralis as the preferred site for muscle biopsy in the evaluation of mitochondrial disease [89].

3. Absence of ragged red fibers and COX-negative fibers does not completely exclude mitochondrial disease.

4. Children under 5 years of age with mitochondria disorders may not have overt ragged red fibers or SDH hyper-reactive fibers. Mild subsarcolemmal mitochondria accumulation is a more common finding in children with mitochondrial myopathy.

5. Myofibers with drying artifact or mild cautery artifact may have selective loss of COX activity, but relatively preserved SDH reactivity. The same is true for atrophic perifascicular fibers in dermatomyositis patients. This abnormality is particularly evident on COX/SDH double stain. In general, any zonal loss of COX reactivity should not be considered evidence of a mitochondria disorder.

\section{Nemaline rods}

Nemaline rods are a feature of nemaline myopathy or sporadic late-onset nemaline myopathy (discussed above, Section III). They are mentioned in this context because they may appear as red subsarcolemmal aggregates on Gomori trichrome stain, or as granular cytoplasmic inclusions on H\&E stain. Nemaline rods may also be seen in other conditions some of which may now be regarded as part of the spectrum of sporadic late-onset nemaline myopathy spectrum including dermatomyositis and HIV [90, 91] and have rarely been reported in normal aging [88].

\section{Ring fibers and tubular aggregates}

Ring fibers are characterized by a peripheral concentric thin layer of myofibrils that appear to encircle a myofiber when viewing the muscle on cross-sections (Fig. 2). Ring fibers are particularly prominent in myotonic dystrophy but may be encountered in other settings.

Tubular aggregates represent inclusions that may be seen as discrete, subsarcolemmal aggregates that are red on the modified Gomori trichrome stain (Fig. 6j). Ultrastructurally these may be composed of stacks of tubules (Fig. 6k), cylindrical spirals (Fig. 61), or vesicular membranous structures (Fig. 6m) [92-94]. They are thought to be derived from sarcoplasmic reticulum and stain on the NADH-TR reaction but lack SDH activity. Tubular aggregates may be seen in some cases of periodic paralysis, some myasthenic syndromes or tubular aggregate myopathy but are also encountered in acquired non-specific myopathies. The finding of tubular aggregates, therefore, requires further correlation with clinical findings and other morphologic changes.

\section{Target and formations and cores}

Target formations are concentric zones of altered myofibrillar arrangement and enzyme content creating a central zone, or bull's eye. They are typically thought of as a feature of neurogenic changes and may be the result of myofiber re-innervation. Morphologically targets show overlap with core formations as discussed above.

\section{Cytoplasmic vacuoles/vacuolar myopathies}

Aggregation of fat, lipids, or membrane-bound vacuoles may all appear as vacuoles by routine light microscopy. The descriptive term "vacuolar myopathy" often provides an initial first step towards arriving at a diagnosis. Subsequent special studies often help to narrow down the differential diagnosis. Periodic acid-Schiff (PAS) staining, oil-red-O staining, acid phosphatase reaction, immunohistochemistry for sarcolemmal proteins, and electron microscopy are helpful tools in defining the nature of inclusions. The rimmed vacuoles of inclusion-body myositis are discussed in Section I. This section will focus on other types of vacuolar myopathies. The main differential considerations include glycogen storage disease (GSD), lipid storage disease (LSD), autophagic vacuolar myopathies, and toxic effects of certain medications.

Vacuole-like spaces are, unfortunately, also a common artifact introduced by suboptimal processing and slow freezing. Ice crystal artifact is common in the frozen section of a muscle biopsy and is often characterized by regional distribution in the biopsy with more and less affected areas. The ice crystals lack peripheral rimming, are optically clear, and tend to be uniformly distributed within the individual affected myofiber, often creating small regularly spaced areas of clearing imparting a sieve like appearance. When there is uncertainty, examining non-frozen specimens resolves the presence of a freezing artifact.

\section{Glycogen storage diseases}

The most common appearance of vacuoles from patients with glycogen storage diseases is that of cytoplasmic 
vacuoles creating subsarcolemmal blebbing (Fig. 6n). These vacuoles contain very fine faint granular material that is often better seen on the modified Gomori trichrome than the H\&E stain. A PAS stain without diastase (Fig. 6o) and electron microscopy confirm the presence of glycogen particles in these blebs. The most common types of glycogen storage disease that may be encountered in a muscle biopsy with subsarcolemmal blebbing are McArdle disease (glycogen storage disease type 5) and adult-onset acid maltase deficiency. McArdle disease is a typical example of a metabolic disease presenting with episodic symptoms, often exercise intolerance and cramping, while patients with adult-onset acid maltase deficiency typically have a slowly progressive clinical course of proximal muscle weakness resembling a muscular dystrophy [95]. The diagnosis of glycogen storage disease type 5 may be confirmed with enzyme histochemistry for myophosphorylase activity, which demonstrates complete absence of staining in myofibers but retained reactivity in endothelial cells (Fig. 6p). The latter is a useful internal control.

Adult-onset acid maltase deficiency results from milder deficiency of the same enzyme that is mutated in Pompe disease (glycogen storage disease type 2). It is the only glycogen storage disease in which glycogen accumulates within lysosomes [96]. The diagnosis of adult-onset acid maltase deficiency may be supported by an acid phosphatase stain to demonstrate the prominence of lysosomes, as well as electron microscopy to confirm the presence of membrane-bound glycogen within lysosomes. Other diseases that interfere with lysosomal function may, however, also have some membrane-bound glycogen. Muscle involvement in adult-onset acid maltase deficiency is quite variable. Paraspinal and respiratory muscles are often prominently affected both clinically and pathologically, while limb muscles may be mildly affected or appear normal morphologically. The earliest finding is increased lysosomal reactivity detectable by acid phosphatase stain, followed by ultrastructural confirmation of glycogen accumulation in membrane-bound lysosomes.

Other rare forms of glycogen storage disease that may demonstrate subsarcolemmal blebbing are type 3 (Cori disease), type 7(Tarui), type 9 (Phosphorylase kinase deficiency), type 10 (Phosphoglycerate mutase deficiency), and type 14 (Phosphoglucomutase 1 deficiency). Glycogen storage disease type 4 (Glycogen debranching enzyme deficiency) may have more structured amylopectin-like deposits called "polyglucosan bodies". Definitive diagnosis of glycogen storage diseases relies on glycolytic pathway enzyme analysis or genetic analysis. It should be noted that normal muscle morphology does not exclude a diagnosis of glycogen storage disease, particularly in a patient with episodic symptoms.

\section{Lipid storage myopathies}

Vacuolar myopathy secondary to lipid storage diseases is generally easy to recognize by morphology. The vacuoles correspond to small round lipid droplets evenly distributed throughout the cytoplasm. These are usually more prominent in type I than type II muscle fibers and may be highlighted by Oil Red O or Sudan Black stains. The most common causes of lipid accumulation are acquired, including malnutrition, total parenteral nutrition, chronic kidney diseases, cirrhosis, drugs, and toxins. Hereditary lipid storage myopathies include primary carnitine deficiency, multiple acyl-CoA dehydrogenase deficiency (MADD) and other beta-oxidation enzyme deficiencies, neutral lipid storage disease with ichthyosis, and neutral lipid storage disease with myopathy [97]. Multi-organ involvement (including liver, brain, and heart) is common in hereditary lipid storage diseases. The classification is difficult on biopsy because of the lack of informative special stains. Definitive diagnosis is usually made by biochemical assays or genetic analysis.

\section{Autophagic vacuolar myopathies}

The two most common forms of autophagic vacuolar myopathies are X-linked myopathy with excessive autophagy (XMEA) and Danon disease. Both show vacuoles that are scattered throughout the cytoplasm rather than clustered in the subsarcolemmal region [98]. The vacuoles are lysosomal in origin and, therefore, positive for acid phosphatase activity like those in adult-onset acid maltase deficiency. They contain glycogen particles and myeloid debris by electron microscopy. These vacuoles also have a membrane with sarcolemmal features, and unlike typical lysosomal membranes, are positive on immunohistochemical stains for sarcolemmal proteins like dystrophin and sarcoglycans [98]. As a result, these disorders have been grouped together as "autophagic vacuolar myopathy with sarcolemmal features". Both, X-linked myopathy with excessive autophagy and Danon disease, are caused by X-linked recessive mutations and the vacuolar myopathy usually manifests only in males. Patients with Danon disease have cardiomyopathy and some have mental retardation. Neither of these two features is typical for patients with $\mathrm{X}$-linked myopathy with excessive autophagy [99]. Danon disease is due to a mutation in the gene encoding lysosomalassociated membrane protein 2 (LAMP-2), and may be confirmed by a negative LAMP-2 immunostain. Distinctive features of X-linked myopathy with excessive autophagy include reduplication of the myofiber basement membrane detected by electron microscopy and intense sarcolemmal deposition of complement C5b-9 [100]. The morphologic diagnosis should be confirmed by genetic analysis. 
Fig. 7 Patterns of muscle atrophy. a Group atrophy and fiber type grouping; the former is evidence of active

denervation, and the latter evidence of re-innervation (biopsy from an adult with acute immune-mediated peripheral neuropathy). b Normal sized fibers with fiber type grouping, indicating prior denervation that has been fully re-innervated. c Group atrophy composed of mixed fiber types suggests active denervation without reinnervation (biopsy from an adult with amyotrophic lateral sclerosis). d Islands of hypertrophic type I fibers and panfascicular atrophy of type II fibers is a characteristic finding in spinal muscular atrophy (biopsy from a 2-year-old infant with spinal muscular atrophy type 2). e Type II atrophy; all type II fibers are small compared to type I fibers. f Early type II atrophy, in which the smallest fibers are type IIb fibers; type IIa fibers are less affected. $\mathbf{g}$ In children, diffuse type I atrophy is a common feature of congenital myopathies (biopsy from a 13-year-old child with central nuclear myopathy). $\mathbf{h}$ In adults, selective type I atrophy is uncommon (biopsy from an adult with myotonic dystrophy). (f ATPase stain at $\mathrm{pH} 4.6$; all remaining panels are ATPase stain at $\mathrm{pH}$ 9.4)
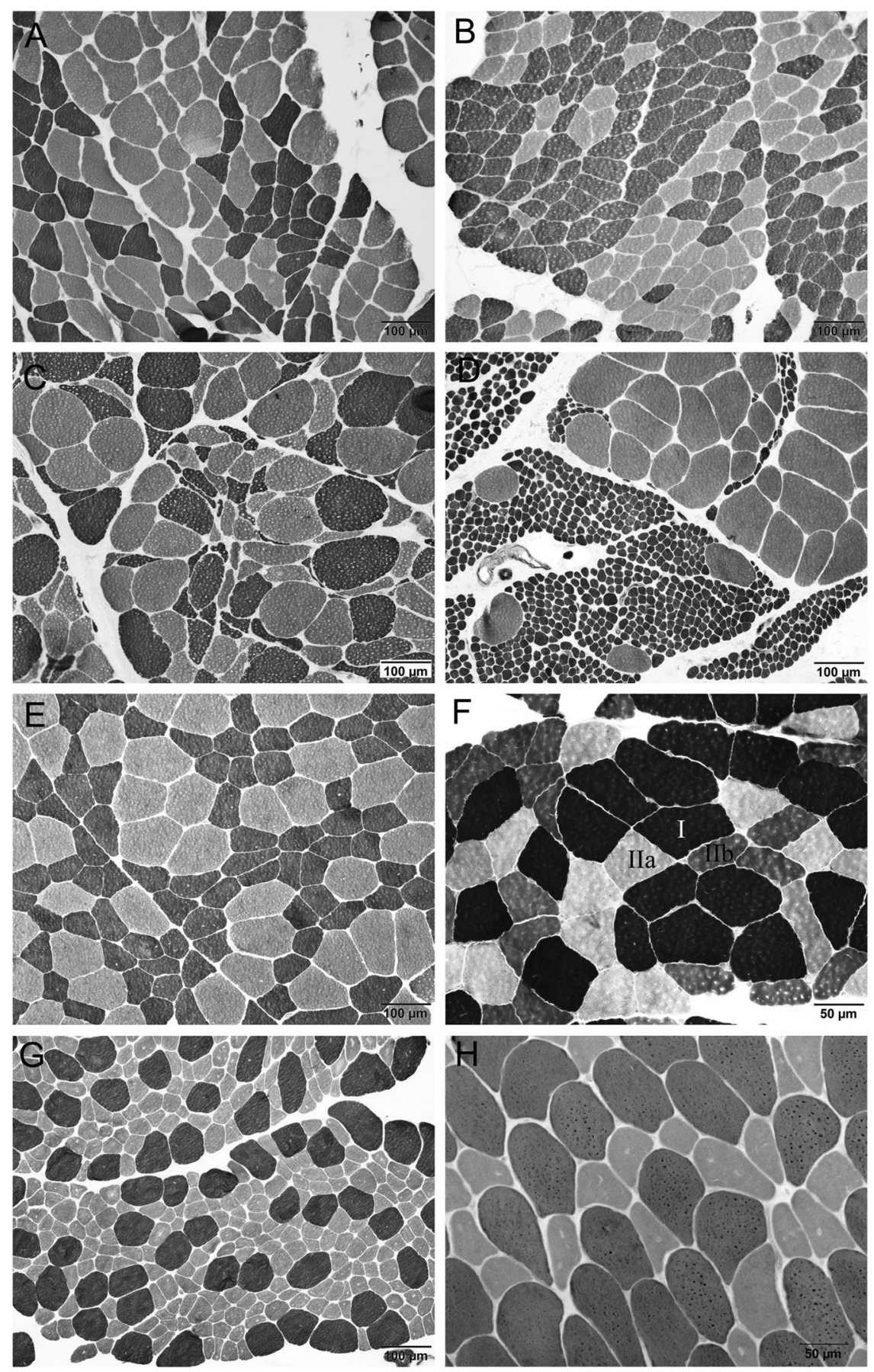

\section{Vacuolar myopathies caused by medication toxicity}

Several medications are known to cause autophagic vacuolar myopathy. Chloroquine and hydroxychloroquine may both lead to myopathy, cardiomyopathy and peripheral neuropathy. Electron microscopy shows cytoplasmic inclusions comprised of whorled lamellar lysosomal debris that may include some glycogen. Identification of curvilinear bodies as also found in neuronal ceroid lipofuscinosis is a helpful diagnostic feature. Colchicine is a common treatment for gout and may also cause vacuolar myopathy affecting primarily type I fibers. Muscle biopsy demonstrates myofibers with central vacuoles positive for acid phosphatase. Ultrastructurally, the vacuoles correspond to membrane-bound membranous material. Vacuolar myopathy has also been reported as a rare complication in patients treated with amiodarone [101]. 


\section{Section V: Biopsies mainly showing atrophic changes}

Myofiber atrophy is seen on biopsies as variability in myofiber size, and is a common morphologic change associated with many different diseases that affect skeletal muscle. Some biopsies may show atrophy as the sole change without associated features of myofiber necrosis and regeneration and without associated inflammatory infiltrates (Fig. 7). It is helpful to recognize certain patterns of muscle atrophy that are associated with distinct disease processes: (a) Grouped atrophy, with clusters of small atrophic, often angulated fibers, is a feature of denervation and is often associated with fiber type grouping as visualized on the ATPase reaction; (b) Perifascicular atrophy is a common feature of dermatomyositis; (c) Selective atrophy of type II fibers may be found in association with disuse, corticosteroid treatment, or endogenous corticosteroid production; (d) Selective atrophy of type I fibers in adults is less common than type II atrophy, but a feature described for example in myotonic dystrophy; (e) In children, selective type I fiber atrophy and predominance of type I fibers are found in most congenital myopathies and myotonic dystrophy type I; (f) Scattered atrophic fibers, randomly affecting fibers of both fiber types, is a common finding in biopsies from patients with neuromuscular symptoms, but may be due to either a neurogenic or myopathic process.

\section{Section VI: Biopsies that appear normal on routine histologic preparations}

Some muscle biopsy specimens may appear normal on the basic histomorphologic studies like H\&E stain, modified Gomori trichrome stain and ATPase reaction. Several explanations should be considered in this setting: (a) The muscle tissue may, indeed, be normal and the patient's symptoms and findings may not be the result of a disorder affecting skeletal muscle. (b) The patient's disease process may not affect the specific muscle that was chosen for biopsy. Some myopathies like adult-onset acid maltase deficiency can result in strikingly selective involvement of certain muscles while sparing others. A common recommendation is for a biopsy to target a moderately affected muscle. (c) The absence of diagnostic changes may be reflective of patchy muscle involvement. Generously sized muscle biopsy specimens sometimes illustrate how some diseases are fairly patchy with abnormalities only seen in some regions of the biopsy. A small sample may, at times, miss the diagnostic features. (d) In rare instances, special studies may be required to identify pathologic changes. Some cases of McArdle disease, carnitine palmitoyltransferase II (CPT-II) deficiency or mitochondrial disease may appear normal by light microscopy. In these cases, enzyme histochemical studies, electron microscopy, biochemical analysis of muscle tissue or genetic testing may be required to tease out the diagnosis. Amyloid deposition within blood vessels or connective tissue is also easily missed without a Congo red stain. In the end, careful review of all clinical information and discussion with the other members of the clinical team are important in cases with normal histomorphology in order to decide which cases warrant further testing.

\section{Conclusion}

We have outlined an approach to neuromuscular pathology based on the common patterns of histomorphologic changes identified on a muscle biopsy. This approach provides the first steps in approaching a biopsy and developing a differential diagnosis. It is difficult to overemphasize the importance of integrating the biopsy findings with pertinent clinical history, physical exam and laboratory findings (Fig. 1). Myopathic changes with inflammation, for example, may fit well for a diagnosis of an inflammatory myopathy in a previously healthy adult with subacute onset of proximal weakness. Similar morphologic features could, however, also be seen in a patient with localized symptoms due to focal myositis or in a patient with a limb girdle muscular dystrophy. A discussion of the results with other members of the clinical team often provides the possibility for more detailed reporting and avoidance for some diagnostic errors. The outlined approach only requires a limited set of initial special stains and may therefore help to use limited health care resources judiciously by avoiding unnecessary special stains that will not contribute to establishing the diagnosis. There have been significant changes in the role that skeletal muscle biopsies have in the workup of patients and in the nature of the clinical questions that accompany these specimens. Often though, skeletal muscle biopsies yield clinically useful information.

\section{Compliance with ethical standards}

Conflict of interest The authors declare that they have no conflict of interest.

\section{References}

1. Domingo-Horne RM, Salajegheh MK. An approach to myopathy for the primary care clinician. Am J Med. 2017; 131:237-43.

2. Gibreel WO, Selcen D, Zeidan MM, Ishitani MB, Moir CR, Zarroug AE. Safety and yield of muscle biopsy in pediatric patients in the modern era. J Pediatr Surg. 2014;49:1429-32.

3. Goutman SA, Prayson RA. Role of repeat skeletal muscle biopsy: how useful is it? Muscle Nerve. 2013;47:835-9. 
4. Lai CH, Melli G, Chang YJ, Skolasky RL, Corse AM, Wagner $\mathrm{KR}$, et al. Open muscle biopsy in suspected myopathy: diagnostic yield and clinical utility. Eur J Neurol. 2010;17:136-42.

5. Nunokawa T, Yokogawa N, Shimada K, Enatsu K, Sugii S. The use of muscle biopsy in the diagnosis of systemic vasculitis affecting small to medium-sized vessels: a prospective evaluation in Japan. Scand J Rheumatol. 2016;45:210-4.

6. Shaibani A, Jabari D, Jabbour M, Arif C, Lee M, Rahbar MH. Diagnostic outcome of muscle biopsy. Muscle Nerve. 2015; 51:662-8.

7. Allenbach Y, Benveniste O. Diagnostic utility of auto-antibodies in inflammatory muscle. Dis J Neuromuscul Dis. 2015;2:13-25.

8. Allenbach Y, Benveniste O, Goebel HH, Stenzel W. Integrated classification of inflammatory myopathies. Neuropathol Appl Neurobiol. 2017;43:62-81.

9. Benveniste O, Stenzel W, Allenbach Y. Advances in serological diagnostics of inflammatory myopathies. Curr Opin Neurol. 2016;29:662-73.

10. Bohan A, Peter JB. Polymyositis and dermatomyositis (second of two parts). N Engl J Med. 1975;292:403-7.

11. Bohan A, Peter JB. Polymyositis and dermatomyositis (first of two parts). N Engl J Med. 1975;292:344-7.

12. Pestronk A. Acquired immune and inflammatory myopathies: pathologic classification. Curr Opin Rheumatol. 2011;23:595-604.

13. Troyanov Y, Targoff IN, Tremblay JL, Goulet JR, Raymond Y, Senecal JL. Novel classification of idiopathic inflammatory myopathies based on overlap syndrome features and autoantibodies: analysis of 100 French Canadian patients. Medicine. 2005;84:231-49.

14. Hoogendijk JE, Amato AA, Lecky BR, Choy EH, Lundberg IE, Rose MR, et al. 119th ENMC international workshop: trial design in adult idiopathic inflammatory myopathies, with the exception of inclusion body myositis, 10-12 October 2003, Naarden, The Netherlands. Neuromuscul Disord. 2004;14:337-45.

15. Troyanov Y, Targoff IN, Payette MP, Raynauld JP, Chartier S, Goulet JR, et al. Redefining dermatomyositis: a description of new diagnostic criteria that differentiate pure dermatomyositis from overlap myositis with dermatomyositis features. Medicine. 2014;93:318-32.

16. van der Meulen MF, Bronner IM, Hoogendijk JE, Burger H, van Venrooij WJ, Voskuyl AE, et al. Polymyositis: an overdiagnosed entity. Neurology. 2003;61:316-21.

17. Lundberg IE, Tjarnlund A, Bottai M, Werth VP, Pilkington C, Visser M, et al. European League Against Rheumatism/American College of Rheumatology classification criteria for adult and juvenile idiopathic inflammatory myopathies and their major subgroups. Ann Rheum Dis. 2017;2017:1955-64.

18. De Bleecker JL, De Paepe B, Aronica E, de Visser M, Amato A, Aronica E, et al. 205th ENMC International Workshop: pathology diagnosis of idiopathic inflammatory myopathies part II 2830 March 2014, Naarden, The Netherlands. Neuromuscul Disord. $2015 ; 25: 268-72$.

19. Suzuki S, Uruha A, Suzuki N, Nishino I. Integrated diagnosis project for inflammatory myopathies: an association between autoantibodies and muscle pathology. Autoimmun Rev. 2017; 16:693-700.

20. Watanabe Y, Uruha A, Suzuki S, Nakahara J, Hamanaka K, Takayama K, et al. Clinical features and prognosis in anti-SRP and anti-HMGCR necrotising myopathy. J Neurol Neurosurg Psychiatry. 2016;87:1038-44.

21. Mescam-Mancini L, Allenbach Y, Hervier B, Devilliers H, Mariampillay K, Dubourg O, et al. Anti-Jo-1 antibody-positive patients show a characteristic necrotizing perifascicular myositis. Brain. 2015;138:2485-92.

22. Stenzel W, Preusse C, Allenbach Y, Pehl D, Junckerstorff R, Heppner FL, et al. Nuclear actin aggregation is a hallmark of anti-synthetase syndrome-induced dysimmune myopathy. Neurology. 2015;84:1346-54.

23. Aguila LA, Lopes MR, Pretti FZ, Sampaio-Barros PD, Carlos de Souza FH, Borba EF, et al. Clinical and laboratory features of overlap syndromes of idiopathic inflammatory myopathies associated with systemic lupus erythematosus, systemic sclerosis, or rheumatoid arthritis. Clin Rheumatol. 2014;33:1093-8.

24. Muro Y, Hosono Y, Sugiura K, Ogawa Y, Mimori T, Akiyama M. Anti-PM/Scl antibodies are found in Japanese patients with various systemic autoimmune conditions besides myositis and scleroderma. Arthritis Res Ther. 2015;17:57.

25. Schmidt J. Current classification and management of inflammatory myopathies. J Neuromuscul Dis. 2018;5:109-29.

26. Blume G, Pestronk A, Frank B, Johns DR. Polymyositis with cytochrome oxidase negative muscle fibres: early quadriceps weakness and poor response to immunosuppressive therapy. Brain. 1997;120(Pt 1):39-45.

27. Levine TD, Pestronk A. Inflammatory myopathy with cytochrome oxidase negative muscle fibers: methotrexate treatment. Muscle Nerve. 1998;21:1724-8.

28. Rifai Z, Welle S, Kamp C, Thornton CA. Ragged red fibers in normal aging and inflammatory myopathy. Ann Neurol. 1995; 37:24-9.

29. Santorelli FM, Sciacco M, Tanji K, Shanske S, Vu TH, Golzi V, et al. Multiple mitochondrial DNA deletions in sporadic inclusion body myositis: a study of 56 patients. Ann Neurol. 1996;39:789-95.

30. Dalakas MC. Inflammatory muscle diseases. N Engl J Med. 2015;372:1734-47.

31. Hiniker A, Daniels BH, Lee HS, Margeta M. Comparative utility of LC3, p62 and TDP-43 immunohistochemistry in differentiation of inclusion body myositis from polymyositis and related inflammatory myopathies. Acta Neuropathol Commun. 2013;1:29.

32. Braczynski AK, Harter PN, Zeiner PS, Drott U, Tews DS, Preusse C, et al. C5b-9 deposits on endomysial capillaries in nondermatomyositis cases. Neuromuscul Disord. 2016;26:283-91.

33. Chahin N, Engel AG. Correlation of muscle biopsy, clinical course, and outcome in PM and sporadic IBM. Neurology. 2008;70:418-24.

34. Nogalska A, Terracciano C, D'Agostino C, King Engel W, Askanas V. p62/SQSTM1 is overexpressed and prominently accumulated in inclusions of sporadic inclusion-body myositis muscle fibers, and can help differentiating it from polymyositis and dermatomyositis. Acta Neuropathol. 2009;118:407-13.

35. Dubourg O, Wanschitz J, Maisonobe T, Behin A, Allenbach Y, Herson S, et al. Diagnostic value of markers of muscle degeneration in sporadic inclusion body myositis. Acta Myol. 2011; 30:103-8.

36. Broccolini A, Mirabella M. Hereditary inclusion-body myopathies. Biochim Biophys Acta. 2015;1852:644-50.

37. Durmus H, Laval SH, Deymeer F, Parman Y, Kiyan E, Gokyigiti M, et al. Oculopharyngodistal myopathy is a distinct entity: clinical and genetic features of 47 patients. Neurology. 2011;76:227-35.

38. Momma K, Noguchi S, Malicdan MC, Hayashi YK, Minami N, Kamakura K, et al. Rimmed vacuoles in Becker muscular dystrophy have similar features with inclusion myopathies. PLoS ONE. 2012;7:e52002.

39. Reilich P, Schramm N, Schoser B, Schneiderat P, Strigl-Pill N, Muller-Hocker J, et al. Facioscapulohumeral muscular dystrophy presenting with unusual phenotypes and atypical morphological features of vacuolar myopathy. J Neurol. 2010;257:1108-18.

40. Semino-Mora C, Dalakas MC. Rimmed vacuoles with betaamyloid and ubiquitinated filamentous deposits in the muscles of patients with long-standing denervation (postpoliomyelitis muscular atrophy): similarities with inclusion body myositis. Hum Pathol. 1998;29:1128-33. 
41. Le Roux K, Streichenberger N, Vial C, Petiot P, Feasson L, Bouhour F, et al. Granulomatous myositis: a clinical study of thirteen cases. Muscle Nerve. 2007;35:171-7.

42. Mozaffar T, Lopate G, Pestronk A. Clinical correlates of granulomas in muscle. J Neurol. 1998;245:519-24.

43. Oxenhandler R, Hart MN, Bickel J, Scearce D, Durham J, Irvin W. Pathologic features of muscle in systemic lupus erythematosus: a biopsy series with comparative clinical and immunopathologic observations. Hum Pathol. 1982;13:745-57.

44. Paik JJ. Myopathy in scleroderma and in other connective tissue diseases. Curr Opin Rheumatol. 2016;28:631-5.

45. Cappelli LC, Gutierrez AK, Bingham CO 3rd, Shah AA. Rheumatic and musculoskeletal immune-related adverse events due to immune checkpoint inhibitors: a systematic review of the literature. Arthritis Care Res. 2017;69:1751-63.

46. Konoeda F, Suzuki S, Nishimoto Y, Hoshino H, Takagi M. A case of myasthenia gravis and myositis induced by nivolumab. Rinsho Shinkeigaku. 2017;57:373-7.

47. Auerbach A, Fanburg-Smith JC, Wang G, Rushing EJ. Focal myositis: a clinicopathologic study of 115 cases of an intramuscular mass-like reactive process. Am J Surg Pathol. 2009;33:1016-24.

48. Ikeda K, Mori-Yoshimura M, Yamamoto T, Sonoo M, Suzuki S, Kondo $\mathrm{Y}$, et al. Chronic myopathy associated with anti-signal recognition particle antibodies can be misdiagnosed as facioscapulohumeral muscular dystrophy. J Clin Neuromuscul Dis. 2016;17:197-206.

49. Mohassel P, Foley AR, Donkervoort S, Fequiere PR, Pak K, Bonnemann CG, et al. Anti-3-hydroxy-3-methylglutaryl-coenzyme a reductase necrotizing myopathy masquerading as a muscular dystrophy in a child. Muscle Nerve. 2017;56:1177-81.

50. Christopher-Stine L, Casciola-Rosen LA, Hong G, Chung T, Corse AM, Mammen AL. A novel autoantibody recognizing 200-kd and $100-\mathrm{kd}$ proteins is associated with an immune-mediated necrotizing myopathy. Arthritis Rheum. 2010;62:2757-66.

51. Mammen AL, Chung T, Christopher-Stine L, Rosen P, Rosen A, Doering KR, et al. Autoantibodies against 3-hydroxy-3-methylglutaryl-coenzyme A reductase in patients with statin-associated autoimmune myopathy. Arthritis Rheum. 2011;63:713-21.

52. Pasnoor M, Barohn RJ, Dimachkie MM. Toxic myopathies. Neurol Clin. 2014;32:647-70. viii

53. Mammen AL. Toxic myopathies. Continuum. 2013;19:1634-49.

54. Mohassel P, Mammen AL. The spectrum of statin myopathy. Curr Opin Rheumatol. 2013;25:747-52.

55. Dalakas MC, Illa I, Pezeshkpour GH, Laukaitis JP, Cohen B, Griffin JL. Mitochondrial myopathy caused by long-term zidovudine therapy. N Engl J Med. 1990;322:1098-105.

56. Fernandez-Sola J, Sacanella E, Estruch R, Nicolas JM, Grau JM, Urbano-Marquez A. Significance of type II fiber atrophy in chronic alcoholic myopathy. J Neurol Sci. 1995;130:69-76.

57. Martin F, Peters TJ. Alcoholic muscle disease. Alcohol Alcohol. 1985;20:125-36.

58. Lilleker JB, Keh YS, Roncaroli F, Sharma R, Roberts M. Metabolic myopathies: a practical approach. Pract Neurol. 2018; 18:14-26.

59. North $\mathrm{KN}$, Wang $\mathrm{CH}$, Clarke $\mathrm{N}$, Jungbluth $\mathrm{H}$, Vainzof $\mathrm{M}$, Dowling JJ, et al. Approach to the diagnosis of congenital myopathies. Neuromuscul Disord. 2014;24:97-116.

60. Colombo I, Scoto M, Manzur AY, Robb SA, Maggi L, Gowda $\mathrm{V}$, et al. Congenital myopathies: Natural history of a large pediatric cohort. Neurology. 2015;84:28-35.

61. Bonnemann CG, Wang $\mathrm{CH}$, Quijano-Roy S, Deconinck N, Bertini E, Ferreiro A, et al. Diagnostic approach to the congenital muscular dystrophies. Neuromuscul Disord. 2014;24:289-311.

62. Selcen D. Myofibrillar myopathies. Neuromuscul Disord. 2011; 21:161-71.
63. Thornton CA. Myotonic dystrophy. Neurol Clin. 2014;32:705-19.

64. Thornton CA, Wang E, Carrell EM. Myotonic dystrophy: approach to therapy. Curr Opin Genet Dev. 2017;44:135-40.

65. Daxinger L, Tapscott SJ, van der Maarel SM. Genetic and epigenetic contributors to FSHD. Curr Opin Genet Dev. 2015;33:56-61.

66. Jungbluth $\mathrm{H}$. Myopathology in times of modern imaging. Neuropathol Appl Neurobiol. 2017;43:24-43.

67. Leung DG. Magnetic resonance imaging patterns of muscle involvement in genetic muscle diseases: a systematic review. J Neurol. 2017;264:1320-33.

68. Morandi L, Mora M, Confalonieri V, Barresi R, Di Blasi C, Brugnoni R, et al. Dystrophin characterization in BMD patients: correlation of abnormal protein with clinical phenotype. J Neurol Sci. 1995;132:146-55.

69. Torelli S, Brown SC, Jimenez-Mallebrera C, Feng L, Muntoni F, Sewry CA. Absence of neuronal nitric oxide synthase (nNOS) as a pathological marker for the diagnosis of Becker muscular dystrophy with rod domain deletions. Neuropathol Appl Neurobiol. 2004;30:540-5.

70. Bello L, Pegoraro E. Genetic diagnosis as a tool for personalized treatment of Duchenne muscular dystrophy. Acta Myol. 2016;35:122-7.

71. Nigro V, Savarese M. Genetic basis of limb-girdle muscular dystrophies: the 2014 update. Acta Myol. 2014;33:1-12.

72. Moore SA, Shilling CJ, Westra S, Wall C, Wicklund MP, Stolle C, et al. Limb-girdle muscular dystrophy in the United States. J Neuropathol Exp Neurol. 2006;65:995-1003.

73. Guglieri M, Magri F, D'Angelo MG, Prelle A, Morandi L, Rodolico C, et al. Clinical, molecular, and protein correlations in a large sample of genetically diagnosed Italian limb girdle muscular dystrophy patients. Hum Mutat. 2008;29:258-66.

74. Norwood FL, Harling C, Chinnery PF, Eagle M, Bushby K, Straub V. Prevalence of genetic muscle disease in Northern England: in-depth analysis of a muscle clinic population. Brain. 2009;132:3175-86.

75. Tarnopolsky MA, Hatcher E, Shupak R. Genetic myopathies initially diagnosed and treated as inflammatory myopathy. Can J Neurol Sci. 2016;43:381-4.

76. Mammen AL. Which nonautoimmune myopathies are most frequently misdiagnosed as myositis? Curr Opin Rheumatol. 2017;29:618-22.

77. Spuler S, Carl M, Zabojszcza J, Straub V, Bushby K, Moore SA, et al. Dysferlin-deficient muscular dystrophy features amyloidosis. Ann Neurol. 2008;63:323-8.

78. Jungbluth H, Treves S, Zorzato F, Sarkozy A, Ochala J, Sewry $\mathrm{C}$, et al. Congenital myopathies: disorders of excitationcontraction coupling and muscle contraction. Nat Rev Neurol. 2018;14:151-67.

79. Jungbluth H, Voermans NC. Congenital myopathies: not only a paediatric topic. Curr Opin Neurol. 2016;29:642-50.

80. Jungbluth H, Lillis S, Zhou H, Abbs S, Sewry C, Swash M, et al. Late-onset axial myopathy with cores due to a novel heterozygous dominant mutation in the skeletal muscle ryanodine receptor (RYR1) gene. Neuromuscul Disord. 2009;19:344-7.

81. Chahin N, Selcen D, Engel AG. Sporadic late onset nemaline myopathy. Neurology. 2005;65:1158-64.

82. Schnitzler LJ, Schreckenbach T, Nadaj-Pakleza A, Stenzel W, Rushing EJ, Van Damme P, et al. Sporadic late-onset nemaline myopathy: clinico-pathological characteristics and review of 76 cases. Orphanet J Rare Dis. 2017;12:86.

83. Preisler N, Lukacs Z, Vinge L, Madsen KL, Husu E, Hansen RS, et al. Late-onset Pompe disease is prevalent in unclassified limbgirdle muscular dystrophies. Mol Genet Metab. 2013;110:287-9.

84. Dimmock DP, Lawlor MW. Presentation and diagnostic evaluation of mitochondrial disease. Pediatr Clin North Am. 2017;64:161-71. 
85. Hasegawa H, Matsuoka T, Goto Y, Nonaka I. Strongly succinate dehydrogenase-reactive blood vessels in muscles from patients with mitochondrial myopathy, encephalopathy, lactic acidosis, and stroke-like episodes. Ann Neurol. 1991;29:601-5.

86. Bernier FP, Boneh A, Dennett X, Chow CW, Cleary MA, Thorburn DR. Diagnostic criteria for respiratory chain disorders in adults and children. Neurology. 2002;59:1406-11.

87. Delisle MB, Laroche M, Dupont H, Rochaix P, Rumeau JL. Morphological analyses of paraspinal muscles: comparison of progressive lumbar kyphosis (camptocormia) and narrowing of lumbar canal by disc protrusions. Neuromuscul Disord. 1993;3:579-82.

88. Tomonaga M. Histochemical and ultrastructural changes in senile human skeletal muscle. J Am Geriatr Soc. 1977;25:125-31.

89. Parikh S, Goldstein A, Koenig MK, Scaglia F, Enns GM, Saneto $\mathrm{R}$, et al. Diagnosis and management of mitochondrial disease: a consensus statement from the Mitochondrial Medicine Society. Genet Med. 2015;17:689-701.

90. Dalakas MC, Pezeshkpour GH, Flaherty M. Progressive nemaline (rod) myopathy associated with HIV infection. N Engl J Med. 1987;317:1602-3.

91. Danon MJ, Giometti CS, Manaligod JR, Perurena OH, Skosey JL. Adult-onset nemaline rods in a patient treated for suspected dermatomyositis. Study with two-dimensional electrophoresis. Arch Neurol. 1981;38:761-6.

92. Jain D, Sharma MC, Sarkar C, Suri V, Sharma SK, Singh S, et al. Tubular aggregate myopathy: a rare form of myopathy. J Clin Neurosci. 2008;15:1222-6.

93. Lee JM, Noguchi S. Calcium dyshomeostasis in tubular aggregate myopathy. Int J Mol Sci. 2016;17:E1952.

94. Chevessier F, Bauche-Godard S, Leroy JP, Koenig J, PaturneauJouas M, Eymard B, et al. The origin of tubular aggregates in human myopathies. J Pathol. 2005;207:313-23.

95. Angelini C. Spectrum of metabolic myopathies. Biochim Biophys Acta. 2015;1852:615-21.

96. Anderson LJ, Henley W, Wyatt KM, Nikolaou V, Waldek S, Hughes DA, et al. Effectiveness of enzyme replacement therapy in adults with late-onset Pompe disease: results from the NCSLSD cohort study. J Inherit Metab Dis. 2014;37:945-52.

97. Liang WC, Nishino I. Lipid storage myopathy. Curr Neurol Neurosci Rep. 2011;11:97-103.

98. Sugie K, Noguchi S, Kozuka Y, Arikawa-Hirasawa E, Tanaka M, Yan C, et al. Autophagic vacuoles with sarcolemmal features delineate Danon disease and related myopathies. J Neuropathol Exp Neurol. 2005;64:513-22.

99. Ramachandran N, Munteanu I, Wang P, Ruggieri A, Rilstone JJ, Israelian N, et al. VMA21 deficiency prevents vacuolar ATPase assembly and causes autophagic vacuolar myopathy. Acta Neuropathol. 2013;125:439-57.

100. Minassian BA, Aiyar R, Alic S, Banwell B, Villanova M, Fardeau M, et al. Narrowing in on the causative defect of an intriguing X-linked myopathy with excessive autophagy. Neurology. 2002;59:596-601.

101. Fernando Roth R, Itabashi H, Louie J, Anderson T, Narahara KA. Amiodarone toxicity: myopathy and neuropathy. Am Heart J. 1990;119:1223-5.

102. Allenbach Y, Leroux G, Suarez-Calvet X, Preusse C, Gallardo E, Hervier B, et al. Dermatomyositis with or without antimelanoma differentiation-associated gene 5 antibodies: common interferon signature but distinct NOS2 expression. Am J Pathol. 2016;186:691-700.

103. Espada G, Maldonado Cocco JA, Fertig N, Oddis CV. Clinical and serologic characterization of an argentine pediatric myositis cohort: identification of a novel autoantibody (anti-MJ) to a 142kDa protein. J Rheumatol. 2009;36:2547-51.

104. Herbert MK, Stammen-Vogelzangs J, Verbeek MM, Rietveld A, Lundberg IE, Chinoy $\mathrm{H}$, et al. Disease specificity of autoantibodies to cytosolic 5'-nucleotidase $1 \mathrm{~A}$ in sporadic inclusion body myositis versus known autoimmune diseases. Ann Rheum Dis. 2016;75:696-701.

105. Ichimura Y, Matsushita T, Hamaguchi Y, Kaji K, Hasegawa M, Tanino Y, et al. Anti-NXP2 autoantibodies in adult patients with idiopathic inflammatory myopathies: possible association with malignancy. Ann Rheum Dis. 2012;71:710-3.

106. Lega JC, Fabien N, Reynaud Q, Durieu I, Durupt S, Dutertre M, et al. The clinical phenotype associated with myositis-specific and associated autoantibodies: a meta-analysis revisiting the so-called antisynthetase syndrome. Autoimmun Rev. 2014;13:883-91.

107. Rigolet A, Musset L, Dubourg O, Maisonobe T, Grenier P, Charuel JL, et al. Inflammatory myopathies with anti-Ku antibodies: a prognosis dependent on associated lung disease. Medicine. 2012;91:95-102.

108. Allenbach Y, Keraen J, Bouvier AM, Jooste V, Champtiaux N, Hervier B, et al. High risk of cancer in autoimmune necrotizing myopathies: usefulness of myositis specific antibody. Brain. 2016;139:2131-5. 